\title{
Conformal covariance of massless free nets
}

\author{
FERNANDO LLEDÓ* \\ Max-Planck-Institut für Gravitationsphysik, \\ Albert-Einstein-Institut, Am Mühlenberg 1, \\ D-14476 Golm, Germany. \\ lledo@aei-potsdam.mpg.de \\ January 18, 2010
}

Dedicated to Hellmut Baumgärtel on the occasion of his 65th birthday.

\begin{abstract}
In the present paper we review in a fibre bundle context the covariant and massless canonical representations of the Poincaré group as well as certain unitary representations of the conformal group (in 4 dimensions). We give a simplified proof of the well-known fact that massless canonical representations with discrete helicity extend to unitary and irreducible representations of the conformal group mentioned before. Further we give a simple new proof that massless free nets for any helicity value are covariant under the conformal group. Free nets are the result of a direct (i.e. independent of any explicit use of quantum fields) and natural way of constructing nets of abstract $\mathrm{C}^{*}$-algebras indexed by open and bounded regions in Minkowski space that satisfy standard axioms of local quantum physics. We also give a group theoretical interpretation of the embedding $\mathfrak{I}$ that completely characterizes the free net: it reduces the (algebraically) reducible covariant representation in terms of the unitary canonical ones. Finally, as a consequence of the conformal covariance we also mention for these models some of the expected algebraic properties that are a direct consequence of the conformal covariance (essential duality, PCT-symmetry etc.).
\end{abstract}

\footnotetext{
* On leave from Mathematical Institute, University of Potsdam, Am Neuen Palais 10, Postfach 601 553, D-14415 Potsdam, Germany.
} 


\section{Introduction}

The birth of massless particles can be traced back to the seminal paper [19 as well as to the most remarkable part of Einstein's famous principle of special relativity also published in 1905 [20] (cf. also [21]): "Wir wollen diese Vermutung (deren Inhalt im folgenden,,Prinzip der Relativität" genannt werden wird) zur Voraussetzung erheben und außerdem die mit ihm nur scheinbar unverträgliche Voraussetzung einführen, daß sich das Licht im leeren Raume stets mit einer bestimmten, vom Bewegungszustande des emittierenden Körpers unabhängigen Geschwindigkeit $V$ fortpflanze." Despite their short history (in comparison with the deeply rooted notion of mass in the physical literature [34]) massless particles are related to several peculiarities in the analysis of the different branches in physics where they enter. For example, extrapolating from the principle above, massless particles inherit a characteristic kinematical behaviour. This aspect of masslessness is used for instance in the corresponding collision theory in quantum field theory (henceforth denoted by QFT): indeed, it is an essential feature of this theory the fact that a massless particle (say at the origin) will be for suitable $t \neq 0$ space-like separated from any point in the interior of the light-cone (cf. [14, 15] and see also [11] for further consequences of the postulate of maximal speed in classical and quantum physics). A different characteristic aspect of masslessness that will be important in this paper appears in Wigner's analysis of the unitary irreducible representations of the Poincaré group [59] (which is the symmetry group of 4-dimensional Minkowski spacetime). Indeed, in this analysis one obtains that the massless little group $\mathcal{E}(2)$ (see (12) ) is noncompact, solvable and has a semi-direct product structure, while the massive little group, $\mathrm{SU}(2)$, satisfies the complementary properties of being compact and simple. Consequences of these differences will obviously only appear for nonscalar models, i.e. in those cases where the corresponding little group is nontrivially represented. For example, in order to get discrete helicity values the solvability and connectedness of $\mathcal{E}(2)$ forces to consider only nonfaithful one-dimensional representations of it, and this fact is related to the physical picture characteristic for $m=0$ that the helicity is relativistic invariant. On the quantum field theoretical side this aspect appears through the need to reduce the degrees of freedom of the fibre of the covariant representation (cf. Section 2 for the group theoretical definitions and the begining of Section 3 for the description of three equivalent ways of performing the reduction). A further characteristic feature of free massless quantum field theoretical models with discrete helicity is that they are covariant w.r.t. the conformal group, i.e. a bigger symmetry group containing as a subgroup the original Poincaré group with which one starts the analysis. In the scalar case (cf. e.g. [36, 32]) one can argue formally that the space of solutions of the wave-equation is invariant under the 
transformation $f \rightarrow R f,(R f)(x):=-\frac{1}{x^{2}} f\left(-\frac{x}{x^{2}}\right)$, where the relativistic ray inversion $x \rightarrow-\frac{x}{x^{2}}$ is one of the generating elements of the conformal group. For higher helicities the conformal covariance of the massless quantum fields still remains true [45, 31] and due to the uniqueness result in [1, 2] (only the unitary irreducible representations of the Poincaré group with $m=0$ and discrete helicity extend within the same Hilbert space to certain unitary representations of the conformal group) it is clear that the reduction of the degrees of freedom mentioned above is an essential feature of the nonscalar models in order to preserve the conformal group as a symmetry group. The conformal covariance will have in its turn remarkable structural consequences for the models. (For a physical interpretation as well as a historical survey on physical applications of the conformal group we refer to [35, 55]).

The intention of the present paper is twofold. On the one hand we review in a fibre bundle context some of the mathematical peculiarities of the unitary and irreducible representations corresponding to $m=0$ and discrete helicity (including a simplified proof of the extension result to a unitary representation of the conformal group). On the other hand we want to give a simple new proof of the fact that in QFT massless models with arbitrary helicity are covariant under the conformal group as well as to apply to these models the important consequences of this covariance. (Here we treat the helicity values as a parameter and no special emphasis is laid on the scalar case.) The simplicity of the proofs mentioned before is partially based on the choice of the notion of a free net in the axiomatic context of 'local quantum physics' (also called algebraic QFT [26, 27]). Free nets as considered in [9, 43] are the result of a direct and natural way of constructing nets of abstract $\mathrm{C}^{*}$-algebras indexed by open and bounded regions in Minkowski space and satisfying Haag-Kastler axioms. The construction is based on group theoretical arguments (concretely on the covariant and canonical representations of the Poincaré group to be introduced in the following section) and standard CAR- or CCR-theory [4. 47]. In the construction no representation of the $\mathrm{C}^{*}$-algebra is used and no quantum fields are explicitly needed and this agrees with the point of view in local quantum physics that the abstract algebraic structure should be a primary definition of the theory and the corresponding Hilbert space representation a secondary [17, Section 4]. In the context of massless models and in particular in gauge quantum field theory this position is not only an esthetic one. Indeed, if constraints are present in the context of bosonic models the use of nonregular representations is sometimes unavoidable at certain stages of the constraint reduction procedure, so that in this frame one is not always allowed to think of the Weyl elements a 'some sort of exponentiated quantum fields' (cf. [24, 22, 25]). Further, the choice of free nets particularly pays off in the 
massless case, since here the use of quantum fields unnecessarily complicates the construction (recall the definition of Weinberg's $2 j+1$-fields that must satisfy the corresponding first-order constraint equation [57, 58, 31]; the necessity of introducing constraints is related to the reduction of the degrees of freedom of the covariant representation mentioned above). Finally, we hope that the study of the mathematical aspects characteristic for massless models will be useful in the analysis of open problems in mathematical physics, where masslessness and nontrivial helicity plays a significant role (e.g. in the context of superselection theory, cf. [16]).

The present paper is structured in 5 sections: in the following section we review in the general frame of induced representations on fibre bundles the covariant and canonical representations of the Poincaré group. We will also point out some of the mathematical differences that appear between the massive and massless canonical representations. Further, we also consider in this context a method to obtain certain unitary representations of the conformal group that will be needed later. In Section 3 we present the definition of a massless free net and state some of its properties, for example they satisfy the Haag-Kastler axioms. The construction is particularly transparent, because of the use of certain reference spaces, where the corresponding sesquilinear form is characterized by positive semidefinite operator-valued functions $\beta(\cdot)$ on the mantle of the forward light-cone $\mathcal{C}_{+}$. The corresponding factor Hilbert spaces (w.r.t. the degenerate subspace) will carry a representation equivalent to the unitary irreducible canonical representation with $m=$ 0 and helicities $\pm \frac{n}{2}$. In the following section we give a simplified proof of the well-known fact that the massless Wigner representations mentioned before extend to certain unitary representations of the conformal group. For the proof the factor space notation of the previous section will be useful. Finally, Section 5 shows the covariance under the conformal group of the massless free nets for any helicity value. Further, using certain natural Fock states and considering the corresponding net of von Neumann algebras we are able to apply the general results stated in [13] for conformal quantum field theories to obtain standard algebraic statements (essential duality, Bisognano-Wichmann Theorem etc.) for these models.

\section{Induced representations: the Poincaré and the conformal group}

In the present section we will summarize some results concerning the theory of induced representations in the context of fibre bundles. For details and further generalizations we refer to [5, 52, 53] and [56, Section 5.1]. We will see below that this general theory beautifully includes all representations of the Poincaré and the conformal group needed in this paper. For further 
aspects of the role played by induced representations in classical and quantum theory see [39] and references cited therein.

Let $\mathcal{G}$ be a Lie group that acts transitively on a $\mathrm{C}^{\infty}$-manifold $M$. Let $u_{0} \in M$ and $\mathcal{K}_{0}:=$ $\left\{g \in \mathcal{G} \mid g u_{0}=u_{0}\right\}$ the corresponding little group w.r.t. this action. Then by [29, Theorem 3.2 and Proposition 4.3] we have that $g \mathcal{K}_{0} \mapsto g u_{0}$ characterizes the diffeomorphism

$$
\mathcal{G} / \mathcal{K}_{0} \cong \mathbb{D}:=\left\{g u_{0} \mid g \in \mathcal{G}\right\}
$$

In this context we may consider the following principal $\mathcal{K}_{0}$-bundle,

$$
\mathcal{B}_{1}:=\left(\mathcal{G}, \operatorname{pr}_{1}, \mathbb{D}\right)
$$

$\mathrm{pr}_{1}: \mathcal{G} \rightarrow \mathbb{D}$ denotes the canonical projection onto the base space $\mathbb{D}$. Given a representation $\tau: \mathcal{K}_{0} \rightarrow \operatorname{GL}(\mathcal{H})$ on the finite-dimensional Hilbert space $\mathcal{H}$, one can construct the associated vector bundle

$$
\mathcal{B}_{2}(\tau):=\left(\mathcal{G} \times_{\mathcal{K}_{0}} \mathcal{H}, \operatorname{pr}_{2}, \mathbb{D}\right)
$$

The action of $\mathcal{G}$ on $M$ specifies the following further actions on $\mathbb{D}$ and on $\mathcal{G} \times_{\mathcal{K}_{0}} \mathcal{H}$ : for $g, g_{0} \in \mathcal{G}$, $v \in \mathcal{H}$, put

$$
\left.\begin{array}{rlrl}
\mathcal{G} \times \mathbb{D} & \longrightarrow \mathbb{D}, & g_{0} \operatorname{pr}_{1}(g):=\operatorname{pr}_{1}\left(g_{0} g\right) \\
\mathcal{G} \times\left(\mathcal{G} \times_{\mathcal{K}_{0}} \mathcal{H}\right) & \longrightarrow \mathcal{G} \times_{\mathcal{K}_{0}} \mathcal{H}, & g_{0}[g, v]:=\left[g_{0} g, v\right],
\end{array}\right\}
$$

where $[g, v]=\left[g k^{-1}, \tau(k) v\right], k \in \mathcal{K}_{0}$, denotes the equivalence class characterizing a point in the total space of the associated bundle. Finally we define the (from $\tau$ ) induced representation of $\mathcal{G}$ on the space of sections of the vector bundle $\mathcal{B}_{2}$, which we denote by $\Gamma\left(\mathcal{G} \times_{\mathcal{K}_{0}} \mathcal{H}\right)$ : let $\psi$ be such a section and for $g \in \mathcal{G}, p \in \mathbb{D}$ :

$$
(T(g) \psi)(p):=g \psi\left(g^{-1} p\right)
$$

2.1 Remark We will now present two ways of rewriting the preceding induced representation in (for physicists more usual) terms of vector-valued functions.

(i) The more standard one consists of choosing a section $s: \mathbb{D} \rightarrow \mathcal{G}$ of the principal $\mathcal{K}_{0}$-bundle $\mathcal{B}_{1}$. Now for $\psi \in \Gamma\left(\mathcal{G} \times_{\mathcal{K}_{0}} \mathcal{H}\right)$ we put $\psi(p)=[s(p), \varphi(p)], p \in \mathbb{D}$, for a suitable function $\varphi: \mathbb{D} \rightarrow \mathcal{H}$ and we may rewrite the induced representation as

$$
(T(g) \varphi)(p)=\tau\left(s(p)^{-1} g s\left(g^{-1} p\right)\right) \varphi\left(g^{-1} p\right)
$$

where it can be easily seen that $s(p)^{-1} g s\left(g^{-1} p\right) \in \mathcal{K}_{0}$. 
(ii) A second less well known way of transcribing the induced representation (国) is done by means of a mapping $J: \mathcal{G} \times \mathbb{D} \rightarrow \mathrm{GL}(\mathcal{H})$ that satisfies

$$
\begin{aligned}
J\left(g_{1} g_{2}, p\right) & =J\left(g_{1}, g_{2} p\right) J\left(g_{2}, p\right), \quad g_{1}, g_{2} \in \mathcal{G}, p \in \mathbb{D} \\
J(e, p) & =\mathbb{1}, \quad \text { where } e \text { is the unit in } \mathcal{G} \\
J\left(k, u_{0}\right) & =\tau(k), \quad k \in \mathcal{K}_{0} .
\end{aligned}
$$

Note that by (66) the l.h.s. of Eq. (8) is indeed a representation of $\mathcal{K}_{0}$. Now for $\psi \in \Gamma\left(\mathcal{G} \times \mathcal{K}_{0} \mathcal{H}\right)$ and a suitable function $\varphi: \mathbb{D} \rightarrow \mathcal{H}$ we may put $\psi(p)=\left[g, J\left(g, u_{0}\right)^{-1} \varphi(p)\right], g \in \mathcal{G}$ and $\operatorname{pr}_{1}(g)=p \in \mathbb{D}$, which is a consistent expression w.r.t. the equivalence classes in $\mathcal{G} \times_{\mathcal{K}_{0}} \mathcal{H}$ : indeed, using (6) and (8) above we have for any $k \in \mathcal{K}_{0}$

$$
\psi(p)=\left[g, J\left(g, u_{0}\right)^{-1} \varphi(p)\right]=\left[g k^{-1}, \tau(k) J\left(g, u_{0}\right)^{-1} \varphi(p)\right]=\left[g k^{-1}, J\left(g k^{-1}, u_{0}\right)^{-1} \varphi(p)\right] .
$$

From this we may rewrite the induced representation as

$$
\left(T\left(g_{0}\right) \varphi\right)(p)=J\left(g_{0}^{-1}, p\right)^{-1} \varphi\left(g_{0}^{-1} p\right), \quad g_{0} \in \mathcal{G}, p \in \mathbb{D} .
$$

Using for example (6)-(8) above it can be directly checked that $T$ is indeed a representation. The present analysis in terms of the mapping $J$ will be useful later in the context of the conformal group (cf. [33, Section I.4]).

Note that till now we have not specified any structure on the sections $\Gamma\left(\mathcal{G} \times_{\mathcal{K}_{0}} \mathcal{H}\right.$ ) (or on the set of $\mathcal{H}$-valued functions). In the following we will apply the preceding general scheme to the Poincaré and the conformal group and will completely fix the structure of the corresponding representation spaces. We will also give regularity conditions on the section $s$ considered in part (i) above.

\subsection{The Poincaré group:}

We will specify next the so-called covariant and canonical representations of the Poincaré group. They will play a fundamental role in the definition of the free net in the next section. Besides the references mentioned at the begining of this section we refer also to [6, 07, 46, 59] as well as 40, Section 2.1].

Covariant representations: In the general analysis considered above let $\mathcal{G}:=\mathrm{SL}(2, \mathbb{C}) \ltimes \mathbb{R}^{4}=$ $\widetilde{\mathcal{P}_{+}^{\uparrow}}$ be the universal covering of the proper orthocronous component of the Poincaré group. It acts on $M:=\mathbb{R}^{4}$ in the usual way $(A, a) x:=\Lambda_{A} x+a,(A, a) \in \mathrm{SL}(2, \mathbb{C}) \ltimes \mathbb{R}^{4}, x \in \mathbb{R}^{4}$, where 
$\Lambda_{A}$ is the Lorentz transformation associated to $\pm A \in \mathrm{SL}(2, \mathbb{C})$ which describes the action of $\mathrm{SL}(2, \mathbb{C})$ on $\mathbb{R}^{4}$ in the last semidirect product. Putting now $u_{0}:=0$ gives $\mathcal{K}_{0}=\mathrm{SL}(2, \mathbb{C}) \ltimes\{0\}$, $\mathcal{G} /(\mathrm{SL}(2, \mathbb{C}) \ltimes\{0\}) \cong \mathbb{R}^{4}$, and the principal $\mathrm{SL}(2, \mathbb{C})$-bundle is in this case $\mathcal{B}_{1}:=\left(\mathcal{G}, \operatorname{pr}_{1}, \mathbb{R}^{4}\right)$. As inducing representation we use the finite-dimensional irreducible representations of $\operatorname{SL}(2, \mathbb{C})$ acting on the spinor space $\mathcal{H}^{\left(\frac{j}{2}, \frac{k}{2}\right)}:=\operatorname{Sym}\left(\stackrel{j}{\otimes} \mathbb{C}^{2}\right) \otimes \operatorname{Sym}\left(\stackrel{k}{\otimes} \mathbb{C}^{2}\right)($ cf. 54$)$ : i.e. $\tau^{(\text {cov })}(A, 0):=$ $D^{\left(\frac{j}{2}, \frac{k}{2}\right)}(A)=(\stackrel{j}{\otimes} A) \otimes(\stackrel{k}{\otimes} \bar{A}),(A, 0) \in \mathrm{SL}(2, \mathbb{C}) \ltimes\{0\}$. From this we have (if no confusion arises we will omit in the following the index $\left(\frac{j}{2}, \frac{k}{2}\right)$ in $D(\cdot)$ and in $\mathcal{H}$ ),

$$
\mathcal{B}_{2}\left(\tau^{\text {(cov) }}\right):=\left(\mathcal{G} \times_{\mathrm{SL}(2, \mathbb{C})} \mathcal{H}, \operatorname{pr}_{2}, \mathbb{R}^{4}\right)
$$

Recalling Remark 2.1 (i) we specify a global continuous section $s$ of $\mathcal{B}_{1}$ (i.e. $\mathcal{B}_{1}$ is a trivial bundle):

$$
s: \mathbb{R}^{4} \longrightarrow \mathcal{G}, \quad s(x):=(\mathbb{1}, x) \in \mathrm{SL}(2, \mathbb{C}) \ltimes \mathbb{R}^{4}=\mathcal{G} .
$$

Note that since $\tau^{(\mathrm{cov})}$ is not a unitary representation and since we want to relate the following socalled covariant representation with the irreducible and unitary canonical ones presented below, it is enough to define $T$ on the space of $\mathcal{H}$-valued Schwartz functions $\mathcal{S}\left(\mathbb{R}^{4}, \mathcal{H}\right)$

$$
(T(g) f)(x):=D(A) f\left(\Lambda_{A}^{-1}(x-a)\right), \quad f \in \mathcal{S}\left(\mathbb{R}^{4}, \mathcal{H}\right)
$$

where we have used that $s(x)^{-1}(A, a) s\left((A, a)^{-1} x\right)=(A, 0),(A, a) \in \mathcal{G} . T$ is an algebraically reducible representation even if the inducing representation $\tau^{(\mathrm{cov})}$ is irreducible.

2.2 Remark In 43, 44] it is shown that the covariant representation is related with the covariant transformation character of quantum fields. Thus a further reason for considering this representation space is the fact that in the heuristic picture we want to smear free quantum fields with test functions in $\mathcal{S}\left(\mathbb{R}^{4}, \mathcal{H}\right)$.

Canonical representations: Next we will consider unitary and irreducible canonical representations of $\widetilde{\mathcal{P}_{+}^{\uparrow}}$ and in particular specify the massless ones with discrete helicity. We will apply in this case Mackey's theory of induced representations of regular semidirect products, where each subgroup is locally compact and one of them abelian [46, 52, ].

First note that in the general context of the begining of this section if $\tau$ is a unitary representation of $\mathcal{K}_{0}$ on $\mathcal{H}$, then $\Gamma\left(\mathcal{G} \times_{\mathcal{K}_{0}} \mathcal{H}\right)$ turns naturally into a Hilbert space. Indeed, the fibres $\operatorname{pr}_{2}^{-1}(p), p \in \mathbb{D}$, inherit a unique (modulo unitary equivalence) Hilbert space structure from $\mathcal{H}$. Assume further that $\mathbb{D}$ allows a $\mathcal{G}$-invariant measure $\mu$. (The following construction goes also through with little modifications if we only require the existence on $\mathbb{D}$ of a quasi-invariant 
measure w.r.t. $\mathcal{G}$.) Then $\Gamma\left(\mathcal{G} \times_{\mathcal{K}_{0}} \mathcal{H}\right)$ is the Hilbert space of all measurable sections $\psi$ of $\mathcal{B}_{2}(\tau)$ that satisfy,

$$
\langle\psi, \psi\rangle=\int_{\mathbb{D}}\langle\psi(p), \psi(p)\rangle_{p} \mu(\mathrm{d} p)<\infty
$$

where $\langle\cdot, \cdot\rangle_{p}$ denotes the scalar product on the Hilbert space $\operatorname{pr}_{2}^{-1}(p), p \in \mathbb{D}$, and the induced representation given in Eq. (雨) is unitary on it.

Put now $\mathcal{G}:=\operatorname{SL}(2, \mathbb{C})$ which acts on $\widehat{\mathbb{R}}^{4}$ by means of the dual action canonically given by the semidirect product structure of $\widetilde{\mathcal{P}_{+}^{\uparrow}}$. It is defined by $\widetilde{\gamma}: \operatorname{SL}(2, \mathbb{C}) \rightarrow \operatorname{Aut} \widehat{\mathbb{R}}^{4}, \chi \in \widehat{\mathbb{R}}^{4}$, and $\left(\widetilde{\gamma}_{A} \chi\right)(a):=\chi\left(\Lambda_{A}^{-1}(a)\right), A \in \mathrm{SL}(2, \mathbb{C}), a \in \mathbb{R}^{4}$. For $\chi \in \widehat{\mathbb{R}}^{4}$ fixed the corresponding little and isotropy subgroups are defined respectively by

$$
\mathcal{G}_{\chi}:=\left\{A \in \operatorname{SL}(2, \mathbb{C}) \mid \widetilde{\gamma}_{A} \chi=\chi\right\}, \mathcal{I}_{\chi}:=\mathcal{G}_{\chi} \ltimes \mathbb{R}^{4} \text { and note that } \widetilde{\mathcal{P}_{+}^{\uparrow}} / \mathcal{I}_{\chi} \cong \mathcal{G} / \mathcal{G}_{\chi} \cong \mathbb{D} \text {. }
$$

We have now the principal $\mathcal{I}_{\chi}$-bundle and the associated bundle given respectively by

$$
\mathcal{B}_{1}:=\left(\widetilde{\mathcal{P}_{+}^{\uparrow}}, \operatorname{pr}_{1}, \mathbb{D}\right) \quad \text { and } \quad \mathcal{B}_{2}\left(\tau^{(\mathrm{can})}\right):=\left(\widetilde{\mathcal{P}_{+}^{\uparrow}} \times_{\mathcal{I}_{\chi}} \mathcal{H}, \mathrm{pr}_{2}, \mathbb{D}\right)
$$

where $\tau^{\text {(can) }}$ is a unitary representation of $\mathcal{I}_{\chi}$ on $\mathcal{H}$. If $\tau^{\text {(can) }}$ is irreducible, then the corresponding induced representation, which is called the canonical representation, is irreducible. Even more, every irreducible representation of $\mathcal{G}$ is obtained (modulo unitary equivalence) in this way. Recall also that the canonical representation is unitary iff $\tau^{(\mathrm{can})}$ is unitary.

To specify massless representations with discrete helicity we choose a character $\chi_{\breve{p}}, \breve{p}:=$ $(1,0,0,1) \in \mathcal{C}_{+}\left(\right.$the mantle of the forward light cone), i.e. $\chi_{\breve{p}}(a)=e^{-i \breve{p} a}, a \in \mathbb{R}^{4}$ and $\breve{p} a$ means the Minkowski scalar product. A straightforward computation shows that the isotropy subgroup is given by $\mathcal{I}_{\chi_{\breve{p}}}=\mathcal{E}(2) \ltimes \mathbb{R}^{4}$, where

$$
\mathcal{E}(2):=\left\{\left(\begin{array}{cc}
e^{\frac{i}{2} \theta} & e^{-\frac{i}{2} \theta} z \\
0 & e^{-\frac{i}{2} \theta}
\end{array}\right) \in \operatorname{SL}(2, \mathbb{C}) \mid \theta \in[0,4 \pi), z \in \mathbb{C}\right\} .
$$

The little group $\mathcal{E}(2)$ is noncompact and since its commutator subgroup is already abelian it follows that $\mathcal{E}(2)$ is solvable. Further, it has again the structure of a semidirect product. (In contrast with this fact we have that the massive little group $\mathrm{SU}(2)$ is compact and simple.) Since $\mathcal{E}(2)$ is a connected and solvable Lie group we know from Lie's Theorem (cf. [7]) that the only finite-dimensional irreducible representations are 1 -dimensional, i.e. $\mathcal{H}:=\mathbb{C}$. Therefore in order to induce irreducible and unitary representations of the whole group that describe discrete helicity values we define

$$
\tau^{(\mathrm{can})}(L, a):=e^{-i \breve{p} a}\left(e^{\frac{i}{2} \theta}\right)^{n}
$$


where $(L, a) \in \mathcal{E}(2) \ltimes \mathbb{R}^{4}=\mathcal{I}_{\chi_{\breve{p}}}, n \in \mathbb{N}$. Note that this representation is not faithful. Indeed, the normal subgroup $\left\{\left(\begin{array}{ll}1 & z \\ 0 & 1\end{array}\right) \mid z \in \mathbb{C}\right\}$ is trivially represented (see also [57, Section II]). Some authors associate this subgroup to certain gauge degrees of freedom of the system (e.g. 28, 37, 51]). We consider next the bundles,

$$
\mathcal{B}_{1}^{\text {(can) }}:=\left(\widetilde{\mathcal{P}_{+}^{\uparrow}}, \operatorname{pr}_{1}, \mathcal{C}_{+}\right) \text {and } \mathcal{B}_{2}\left(\tau^{\text {(can) }}\right):=\left(\widetilde{\mathcal{P}_{+}^{\uparrow}} \times_{\mathcal{I}_{\chi_{\breve{p}}}} \mathbb{C}, \operatorname{pr}_{2}, \mathcal{C}_{+}\right)
$$

where we have used the diffeomorphism $\widetilde{\mathcal{P}_{+}^{\uparrow}} / \mathcal{I}_{\chi_{\breve{p}}} \cong \mathcal{C}_{+}$between the factor space and the mantle of the forward light-cone. We denote by $\mu_{0}(\mathrm{~d} p)$ the corresponding invariant measure on $\mathcal{C}_{+}$.

In contrast with the massive case the bundle $\mathcal{B}_{1}^{(\mathrm{can})}$ has no global continuous section. This fact is based on the comparison of different homotopy groups that can be associated with the bundle $\mathcal{B}_{1}^{(\mathrm{can})}$ 12. Nevertheless, we can specify a measurable section considering a continuous one in a chart that does not include the set $\left\{p \in \mathcal{C}_{+} \mid p_{3}=-p_{0}\right\}$ (which is of measure zero w.r.t. $\mu_{0}(\mathrm{~d} p)$ ). Putting $\mathcal{C}_{+}^{\circ}:=\mathcal{C}_{+} \backslash\left\{p \in \mathcal{C}_{+} \mid p_{3}=-p_{0}\right\}$ a (local) continuous section is given explicitly by

$$
s: \mathcal{C}_{+}^{\circ} \longrightarrow \widetilde{\mathcal{P}_{+}^{\uparrow}}, \quad s(p):=\left(H_{p}, 0\right) \in \mathrm{SL}(2, \mathbb{C}) \ltimes \mathbb{R}^{4}=\widetilde{\mathcal{P}_{+}^{\uparrow}},
$$

where

$$
H_{p}:=\frac{1}{\sqrt{2 p_{0}\left(p_{0}+p_{3}\right)}}\left(\begin{array}{cc}
-\sqrt{p_{0}}\left(p_{0}+p_{3}\right) & \frac{p_{1}-i p_{2}}{\sqrt{p_{0}}} \\
-\sqrt{p_{0}}\left(p_{1}+i p_{2}\right) & -\frac{p_{0}+p_{3}}{\sqrt{p_{0}}}
\end{array}\right) .
$$

Recall that the $H_{p}$-matrices satisfy the equation

$$
H_{p}\left(\begin{array}{ll}
2 & 0 \\
0 & 0
\end{array}\right) H_{p}^{*}=P, \quad \text { where } P=\left(\begin{array}{cc}
p_{0}+p_{3} & p_{1}-i p_{2} \\
p_{1}+i p_{2} & p_{0}-p_{3}
\end{array}\right)=p_{0} \sigma_{0}+\sum_{i=1}^{3} p_{i} \sigma_{i}
$$

where $\sigma_{\mu}, \mu=0,1,2,3$, are the unit and the Pauli matrices and we have used the vector space isomorphism between $\mathbb{R}^{4}$ and $\mathrm{H}(2, \mathbb{C}):=\left\{P \in \operatorname{Mat}_{2}(\mathbb{C}) \mid P^{*}=P\right\}$ given by $\mathbb{R}^{4} \ni p:=\left(p_{0}, p_{1}, p_{2}, p_{3}\right) \mapsto P$.

If we consider the section in Eq. (14) fixed, then we have on $\mathrm{L}^{2}\left(\mathcal{C}_{+}, \mathbb{C}, \mu_{0}(\mathrm{~d} p)\right)$ the canonical massless representations (cf. Eq. (5))

$$
\left(U_{ \pm}(g) \varphi\right)(p)=e^{-i p a}\left(e^{ \pm \frac{i}{2} \theta(A, p)}\right)^{n} \varphi(q)
$$

where $g=(A, a) \in \mathrm{SL}(2, \mathbb{C}) \ltimes \mathbb{R}^{4}, n \in \mathbb{N}, q:=\Lambda_{A}^{-1} p$ and for $A=\left(\begin{array}{ll}a & b \\ c & d\end{array}\right) \in \mathrm{SL}(2, \mathbb{C})$ we compute

$$
e^{-\frac{i}{2} \theta(A, p)}:=\left(H_{p}^{-1} A H_{q}\right)_{22}=\frac{-b\left(p_{1}+i p_{2}\right)+d\left(p_{0}+p_{3}\right)}{\left|-b\left(p_{1}+i p_{2}\right)+d\left(p_{0}+p_{3}\right)\right|} .
$$

$U_{ \pm}$are unitary w.r.t. usual $\mathrm{L}^{2}$-scalar product, satisfy the spectrum condition and the helicity of the model carrying one of these representations is $\pm \frac{n}{2}$. 


\subsection{The conformal group:}

We will consider first some standard facts concerning the conformal group [30, Appendix], [50, 55, 33]. We will describe later a technique to define a unitary representation of $\mathrm{SU}(2,2)$, by means of the mapping $J$ considered in Remark 2.1 (ii). These results are a variation of the notion of reproducing kernel for which we refer to [38, 33, 18] and will be useful in order to extend the massless canonical representations to unitary representations of the conformal group.

The group

$$
\mathrm{SU}(2,2):=\left\{g \in \operatorname{Mat}_{4}(\mathbb{C}) \mid \operatorname{det} g=1 \text { and } g \zeta g^{*}=\zeta\right\}, \quad \text { where } \zeta:=\left(\begin{array}{cc}
0 & -i \mathbb{1} \\
i \mathbb{1} & 0
\end{array}\right) \text {, }
$$

is the fourfold covering of the conformal group in Minkowski space. Using $A, B, C, D \in \operatorname{Mat}_{2}(\mathbb{C})$ we have that $g=\left(\begin{array}{ll}A & B \\ C & D\end{array}\right) \in \mathrm{SU}(2,2)$ iff $\operatorname{det} g=1$ and

$$
\left.\begin{array}{ccl}
A B^{*}=B A^{*} & & C^{*} A=A^{*} C \\
C D^{*}=D C^{*} & \text { or equivalently } & B^{*} D=D^{*} B \\
D^{*}-B C^{*}=\mathbb{1} & & A^{*} D-C^{*} B=\mathbb{1}
\end{array}\right\}
$$

Further, we write the natural action of $\mathrm{SU}(2,2)$ on the forward tube

$$
\mathbb{T}_{+}:=\mathrm{H}(2, \mathbb{C})+i \mathrm{H}_{+}(2, \mathbb{C}) \cong \mathbb{R}^{4}+i \mathcal{V}_{+}, \quad \mathrm{H}_{+}(2, \mathbb{C}):=\{P \in \mathrm{H}(2, \mathbb{C}) \mid \operatorname{det} P>0, \operatorname{Tr} P>0\}
$$

as follows:

$$
g Z:=(A Z+B)(C Z+D)^{-1}, \quad Z=X+i Y \in \mathbb{T}_{+} .
$$

Finally, the Poincaré group, the dilations and the special conformal transformations can be recovered as subgroups of $\mathrm{SU}(2,2)$. In particular we will need later

$$
\widetilde{\mathcal{P}_{+}^{\uparrow}}=\left\{\left(\begin{array}{cc}
A & B\left(A^{*}\right)^{-1} \\
0 & \left(A^{*}\right)^{-1}
\end{array}\right) \mid A \in \mathrm{SL}(2, \mathbb{C}), B \in \mathrm{H}(2, \mathbb{C})\right\} \subset \mathrm{SU}(2,2) .
$$

With this action in mind recall the general situation concerning induced representations at the begininng of this section and put now $\mathcal{G}:=\mathrm{SU}(2,2), M:=\mathbb{T}_{+}$and $u_{0}:=i \mathbb{1} \in \mathbb{T}_{+}$, so that from the action given in Eq. (20) we get [33, Section 3]

$$
\mathcal{K}_{0}:=\{g \in \mathrm{SU}(2,2) \mid g i \mathbb{1}=i \mathbb{1}\}=\left\{\left(\begin{array}{cc}
A & B \\
-B & A
\end{array}\right) \in \mathrm{SU}(2,2)\right\} \quad \text { and } \mathrm{SU}(2,2) / \mathcal{K}_{0} \cong \mathbb{T}_{+} .
$$


Suppose now that there exists a Hilbert space $\mathfrak{H}$ with scalar product $\langle\cdot, \cdot\rangle$ (we will identify later $\mathfrak{H}$ with the representation Hilbert space of the massless canonical representations) and that we may use $\mathbb{T}_{+}$and $\mathcal{H}$ to parametrize a total set

$$
\mathfrak{H}_{\text {tot }}:=\left\{K_{z, v} \mid z \in \mathbb{T}_{+}, v \in \mathcal{H}\right\} \subset \mathfrak{H} .
$$

2.3 Lemma If the scalar product satisfies on $\mathfrak{H}_{\text {tot }}$ the property

$$
\left\langle K_{g z_{1}, v_{1}}, K_{g z_{2}, v_{2}}\right\rangle=\left\langle K_{z_{1}, J\left(g, z_{1}\right)^{*} v_{1}}, K_{z_{2}, J\left(g, z_{2}\right)^{*} v_{2}}\right\rangle, \quad z_{1}, z_{2} \in \mathbb{T}_{+}, v_{1}, v_{2} \in \mathcal{H}
$$

for all $g \in \mathrm{SU}(2,2)$, then the representation defined on $\mathfrak{H}_{\text {tot }}$ by

$$
V(g) K_{z, v}:=K_{g z,\left(J(g, z)^{-1}\right)^{*} v} \in \mathfrak{H}_{\text {tot }}
$$

extends to a unitary representation within $\mathfrak{H}$.

Proof: First of all note that on $\mathfrak{H}_{\text {tot }}$ the relation $V\left(g_{1} g_{2}\right)=V\left(g_{1}\right) V\left(g_{2}\right), g_{1}, g_{2} \in \mathrm{SU}(2,2)$, holds. Indeed, using Eq. (6) we have

$$
V\left(g_{1} g_{2}\right) K_{z, v}=K_{g_{1} g_{2} z,\left(J\left(g_{1} g_{2}, z\right)^{-1}\right)^{*} v}=K_{g_{1} g_{2} z,\left(J\left(g_{1}, g_{2} z\right)^{-1}\right)^{*}\left(J\left(g_{2}, z\right)^{-1}\right)^{*} v}=V\left(g_{1}\right)\left(V\left(g_{2}\right) K_{z, v}\right) .
$$

We can also easily check the isometry property on span $\mathfrak{H}_{\text {tot }}$, which by assumption is dense in $\mathfrak{H}$.

For $\sum_{l=1}^{L} \lambda_{l} K_{z_{l}, v_{l}}, \sum_{m=1}^{M} \lambda_{m}^{\prime} K_{z_{m}^{\prime}, v_{m}^{\prime}} \in \operatorname{span} \mathfrak{H}_{t o t}$ and extending by linearity the above definition we have

$$
\begin{aligned}
\left\langle V(g)\left(\sum_{l=1}^{L} \lambda_{l} K_{z_{l}, v_{l}}\right), V(g)\left(\sum_{m=1}^{M} \lambda_{m}^{\prime} K_{z_{m}^{\prime}, v_{m}^{\prime}}\right)\right\rangle & \\
= & \sum_{l, m} \overline{\lambda_{l}} \lambda_{m}^{\prime}\left\langle K_{g z_{l},\left(J\left(g, z_{l}\right)^{-1}\right)^{*} v_{l}}, K_{g z_{m}^{\prime},\left(J\left(g, z_{m}^{\prime}\right)^{-1}\right)^{*} v_{m}^{\prime}}\right\rangle \\
= & \sum_{l, m} \overline{\lambda_{l}} \lambda_{m}^{\prime}\left\langle K_{z_{l}, J\left(g, z_{l}\right)^{*}\left(J\left(g, z_{l}\right)^{-1}\right)^{*} v_{l}}, K_{z_{m}^{\prime}, J\left(g, z_{m}^{\prime}\right)^{*}\left(J\left(g, z_{m}^{\prime}\right)^{-1}\right)^{*} v_{m}^{\prime}}\right\rangle \\
= & \left\langle\sum_{l=1}^{L} \lambda_{l} K_{z_{l}, v_{l}}, \sum_{m=1}^{M} \lambda_{m}^{\prime} K_{z_{m}^{\prime}, v_{m}^{\prime}}\right\rangle .
\end{aligned}
$$

We can therefore extend isometrically $V(g)$ to a unitary representation on the whole $\mathfrak{H}$.

\section{Massless free nets}

In the following we will briefly review with some modifications and improvements the massless free net construction presented in [9, Part B]. The fundamental object that characterizes a free 
net is the linear embedding $\mathfrak{I}$ that intertwines between the covariant and the canonical representation. The free net will be called massive or massless depending if the canonical representation corresponds to $m>0$ resp. $m=0$. Now a typical feature of massless models with helicity $\neq 0$ is the fact that the embeddings must reduce the degrees of freedom on the fibres of the corresponding associated bundles. Indeed, as a consequence of the fact that $\mathcal{E}(2)$ is solvable we have that the fibres of $\mathcal{B}_{2}\left(\tau^{\text {(can) }}\right)$ are 1 -dimensional, while the fibres of $\mathcal{B}_{2}\left(\tau^{\text {(cov) }}\right)$ are at least 2 -dimensional if one chooses a nontrivial inducing representation $\tau^{\text {(cov) }}$. With other words, if the models describe nontrivial helicity, then some further restriction must be performed on the fibres in order to reduce the covariant representation to the unitary and irreducible canonical one. There are at least three ways to perform the mentioned reduction that will produce isomorphic nets of $\mathrm{C}^{*}$-algebras:

(i) One possibility that will be considered next is to rewrite the massless canonical representation in a for us much more convenient way. Using certain natural reference spaces with a semidefinite sesquilinear form characterized by an positive semidefinite operator-valued function $\beta(\cdot)$, the reduction is done passing to the factor spaces that can be canonically constructed from the degeneracy subspaces of the sesquilinear form.

(ii) A second possibility is to consider other type of embeddings that map the Schwartz test functions to the space of solutions of the corresponding massless relativistic wave equations. Here the reduction is done by means of certain invariant (but not reducing) projections on the spinor space $\mathcal{H}$ (cf. 43, 44]).

(iii) Finally, one can also perform the mentioned reduction for the bosonic models at the $\mathrm{C}^{*}$ level by the constraint reduction procedure of Grundling and Hurst [23]. In this context the constraints can be defined as the Weyl elements associated to the degenerate subspace of part (i) (cf. 442) and the constraint reduction here is similar to the second stage of reduction of the Gupta-Bleuler model considered in [25, Theorem 5.14].

The essence of the following construction is the fact that for each $p \in \mathcal{C}_{+}$the nonnegative, selfadjoint matrix $P^{\dagger}=\frac{1}{2}\left(\begin{array}{cc}p_{0}-p_{3} & -p_{1}+i p_{2} \\ -p_{1}-i p_{2} & p_{0}+p_{3}\end{array}\right)$ has the eigenvalues $p_{0}$ and 0 :

$$
P^{\dagger}\left(\begin{array}{c}
-p_{1}+i p_{2} \\
p_{0}+p_{3}
\end{array}\right)=p_{0}\left(\begin{array}{c}
-p_{1}+i p_{2} \\
p_{0}+p_{3}
\end{array}\right) \quad \text { and } \quad P^{\dagger}\left(\begin{array}{c}
p_{1}-i p_{2} \\
p_{0}-p_{3}
\end{array}\right)=0\left(\begin{array}{c}
p_{1}-i p_{2} \\
p_{0}-p_{3}
\end{array}\right) \text {. }
$$

That $P^{\dagger}$ has the eigenvalue 0 is a typical feature of massless representations, since for the massive ones the corresponding matrix $P^{\dagger}$ is strictly positive for any $p$ on the positive mass shell (see [9, 
Part A]). Now each function $\varphi: \mathcal{C}_{+} \backslash\left\{p \in \mathcal{C}_{+}|| p_{3} \mid=p_{0}\right\} \longrightarrow \mathbb{C}^{2}$ can be decomposed pointwise into a sum of the eigenvectors above (recall that $\left\{p \in \mathcal{C}_{+}|| p_{3} \mid=p_{0}\right\}$ is of measure 0 w.r.t $\mu_{0}$ ),

$$
\varphi(p)=\left(\begin{array}{c}
-p_{1}+i p_{2} \\
p_{0}+p_{3}
\end{array}\right) \alpha_{+}(p)+\left(\begin{array}{c}
p_{1}-i p_{2} \\
p_{0}-p_{3}
\end{array}\right) \alpha_{0}(p)
$$

for suitable $\mathbb{C}$-valued functions $\alpha_{+}, \alpha_{0}$. Further, the matrix $P^{\dagger}$ is a natural object from the point of view of representation theory of the Poincaré group. It is straightforward to show that $P^{\dagger}=\left(H_{p}^{-1}\right)^{*}\left(\begin{array}{ll}0 & 0 \\ 0 & 1\end{array}\right) H_{p}^{-1}=\frac{1}{2}\left(p_{0} \sigma_{0}-\sum_{i=1}^{3} p_{i} \sigma_{i}\right)$, where the matrices $H_{p} \in \mathrm{SL}(2, \mathbb{C}), p \in \mathcal{C}_{+}^{\circ}$, are given in Eq. (15).

The sesquilinear forms to be defined next are characterized by the following positive semidefinite operator-valued functions: put for $p \in \mathcal{C}_{+}$and $n \in \mathbb{N}$,

$$
\beta_{+}(p):=D^{\left(0, \frac{n}{2}\right)}\left(P^{\dagger}\right)=\stackrel{n}{\otimes} \overline{P^{\dagger}} \quad \text { and } \quad \beta_{-}(p):=D^{\left(\frac{n}{2}, 0\right)}\left(P^{\dagger}\right)=\stackrel{n}{\otimes} P^{\dagger}
$$

$\beta_{ \pm}(p)$ act on $\mathcal{H}^{\left(0, \frac{n}{2}\right)}$ resp. $\mathcal{H}^{\left(\frac{n}{2}, 0\right)}$. Define then for $\varphi, \psi$ a pair of $\mathcal{H}$-valued measurable functions the sesquilinear forms

$$
\langle\varphi, \psi\rangle_{\beta_{ \pm}}:=\int_{\mathcal{C}_{+}}\left(\varphi(p), \beta_{ \pm}(p) \psi(p)\right)_{\mathcal{H}} \mu_{0}(\mathrm{~d} p),
$$

and from this consider the sets

$$
\mathfrak{H}_{n, \pm}:=\left\{\varphi: \mathcal{C}_{+}^{\circ} \longrightarrow \mathcal{H} \mid \varphi \text { is measurable and }\langle\varphi, \varphi\rangle_{\beta_{ \pm}}<\infty\right\}
$$

For $\varphi_{ \pm} \in \mathfrak{H}_{n, \pm}$ we define also the representations:

$$
\begin{aligned}
& \left(V_{1}(g) \varphi_{+}\right)(p):=e^{-i p a} D^{\left(0, \frac{n}{2}\right)}(A) \varphi_{+}(q), \\
& \left(V_{2}(g) \varphi_{-}\right)(p):=e^{-i p a} D^{\left(\frac{n}{2}, 0\right)}(A) \varphi_{-}(q),
\end{aligned}
$$

where $g=(A, a) \in \widetilde{\mathcal{P}_{+}^{\uparrow}}=\operatorname{SL}(2, \mathbb{C}) \ltimes \mathbb{R}^{4}$ and $q:=\Lambda_{A}^{-1} p \in \mathcal{C}_{+}$. Since

$$
\beta_{+}(q)=D^{\left(0, \frac{n}{2}\right)}(A)^{*} \beta_{+}(p) D^{\left(0, \frac{n}{2}\right)}(A) \quad \text { and } \quad \beta_{-}(q)=D^{\left(\frac{n}{2}, 0\right)}(A)^{*} \beta_{-}(p) D^{\left(\frac{n}{2}, 0\right)}(A)
$$

for $p, q$ as before we have that the representations $V_{1,2}$ leave the sesquilinear forms $\langle\cdot, \cdot\rangle_{\beta_{ \pm}}$invariant. From the comments made at the beginning of this section about the eigenvalues of $P^{\dagger}$ it is clear that the sesquilinear forms $\langle\cdot, \cdot\rangle_{\beta_{ \pm}}$are only semidefinite. This observation is in agreement with the general theorem in [6, p. 113]. We can thus select in a natural way the following subspaces of $\mathfrak{H}_{n, \pm}$ : 
3.1 Definition With respect to the sesquilinear form defined above we can naturally define:

$$
\begin{aligned}
\mathfrak{H}_{n,+}^{(>)} & :=\left\{\varphi \in \mathfrak{H}_{n,+} \mid \varphi(p)=\stackrel{n}{\otimes}\left(\begin{array}{c}
-p_{1}-i p_{2} \\
p_{0}+p_{3}
\end{array}\right) \chi_{+}(p), \text { for suitable scalar } \chi_{+}\right\} \\
\mathfrak{H}_{n,-}^{(>)} & :=\left\{\varphi \in \mathfrak{H}_{n,-} \mid \varphi(p)=\stackrel{n}{\otimes}\left(\begin{array}{c}
-p_{1}+i p_{2} \\
p_{0}+p_{3}
\end{array}\right) \chi_{-}(p), \text { for suitable scalar } \chi_{-}\right\} \\
\mathfrak{H}_{n, \pm}^{(0)} & :=\left\{\varphi \in \mathfrak{H}_{n, \pm} \mid\langle\varphi, \varphi\rangle_{\beta_{ \pm}}=0\right\} \\
\mathfrak{H}_{n, \pm}^{\prime} & :=\mathfrak{H}_{n, \pm} / \mathfrak{H}_{n, \pm}^{(0)}
\end{aligned}
$$

3.2 Lemma Using the preceding definitions we have that for $n>0$

(i) $\mathfrak{H}_{n, \pm}=\mathfrak{H}_{n, \pm}^{(>)} \oplus \mathfrak{H}_{n, \pm}^{(0)}$.

(ii) The representations $V_{1,2}$ leave the spaces $\mathfrak{H}_{n, \pm}^{(0)}$ invariant. On the contrary, the subspaces $\mathfrak{H}_{n, \pm}^{(>)}$are not invariant under the mentioned representations.

(iii) For any non zero $\varphi \in \mathfrak{H}_{n, \pm}^{(>)}$we have $\|\varphi\|_{\beta_{ \pm}}=\left\|V_{1,2}(g) \varphi\right\|_{\beta_{ \pm}}>0$ for all $g \in \widetilde{\mathcal{P}_{+}^{\uparrow}}$.

Proof: Part (i) follows directly from the analysis of the eigenvalues of the matrix $P^{\dagger}$ given at the beginning of this section. That $\mathfrak{H}_{n, \pm}^{(0)}$ are $V_{1,2}$-invariant subspaces and part (iii) are a consequence of the fact that the representations $V_{1,2}$ leave the sesquilinear forms $\langle\cdot, \cdot\rangle_{\beta_{ \pm}}$invariant. To prove the rest of part (ii) note that e.g. for $f \in \mathcal{S}\left(\mathbb{R}^{4}, \mathcal{H}\right)$ we have

$$
\varphi_{+}^{(>)}(p):=D^{\left(0, \frac{n}{2}\right)}\left(H_{p}\left(\begin{array}{ll}
0 & 0 \\
0 & 1
\end{array}\right)\right) \widehat{f}(p) \in \mathfrak{H}_{n,+}^{(>)} \quad \text { and } \quad \varphi_{+}^{(0)}(p):=D^{\left(0, \frac{n}{2}\right)}\left(H_{p}\left(\begin{array}{ll}
1 & 0 \\
0 & 0
\end{array}\right)\right) \widehat{f}(p) \in \mathfrak{H}_{n,+}^{(0)} .
$$

Thus for a general $g=(A, 0) \in \widetilde{\mathcal{P}_{+}^{\uparrow}}$ and since $H_{p}^{-1} A H_{q} \in \mathcal{E}(2), q:=\Lambda_{A}^{-1} p$,

$$
\left(V_{1}(g) \varphi_{+}^{(>)}\right)(p)=D^{\left(0, \frac{n}{2}\right)}\left(A H_{q}\left(\begin{array}{ll}
0 & 0 \\
0 & 1
\end{array}\right)\right) \hat{f}(q)=D^{\left(0, \frac{n}{2}\right)}\left(H_{p}\right) D^{\left(0, \frac{n}{2}\right)}\left(H_{p}^{-1} A H_{q}\left(\begin{array}{ll}
0 & 0 \\
0 & 1
\end{array}\right)\right) \hat{f}(q)=\psi_{+}^{(>)}+\psi_{+}^{(0)},
$$

where $\psi_{+}^{(>)} \in \mathfrak{H}_{n,+}^{(>)}, \psi_{+}^{(0)} \in \mathfrak{H}_{n,+}^{(0)}$ (similar arguments for the spaces with opposite helicity indexed with a '-'). This implies that the representations $V_{1,2}$ restricted to $\mathfrak{H}_{n, \pm}^{(>)}$produce in general further 'zero norm vectors'.

From the preceding lemma we can lift the representations $V$ to the factor spaces $\mathfrak{H}_{n, \pm}^{\prime}$. We denote the lift by $V^{\prime}$ and the equivalence classes in $\mathfrak{H}_{n, \pm}^{\prime}$ by $[\cdot]_{ \pm}$.

3.3 Theorem The representations $V_{1,2}^{\prime}$ defined on $\mathfrak{H}_{n, \pm}^{\prime}$ are equivalent to the irreducible and unitary Wigner representations $U_{ \pm}$defined in Eq. (17). 
Proof: We will give the proof for the spaces with index ' + '. For the spaces with opposite helicity similar arguments can be used just interchanging $D^{\left(0, \frac{n}{2}\right)}(\cdot)$ with $D^{\left(\frac{n}{2}, 0\right)}(\cdot)$. For $\chi \in$ $\mathrm{L}^{2}\left(\mathcal{C}_{+}, \mathbb{C}, \mu_{0}(\mathrm{~d} p)\right)$ the linear mapping given by

$$
\left(\Phi_{+} \chi\right)(p):=\left[D^{\left(0, \frac{n}{2}\right)}\left(H_{p}\right)\left(\stackrel{n}{\otimes}\left(\begin{array}{l}
0 \\
1
\end{array}\right)\right) \chi(p)\right]_{+}
$$

is easily seen to be an isometry between $\mathrm{L}^{2}\left(\mathcal{C}_{+}, \mathbb{C}, \mu_{0}(\mathrm{~d} p)\right)$ and $\mathfrak{H}_{n,+}^{\prime}$ with the corresponding scalar products. Note that $D^{\left(0, \frac{n}{2}\right)}\left(H_{p}\right)\left(\stackrel{n}{\otimes}\left(\begin{array}{l}0 \\ 1\end{array}\right)\right) \chi(p)$ is the representant in $\mathfrak{H}_{n,+}^{(>)}$of the equivalence class. Now the intertwining equation $\Phi_{+} U_{+}(g)=V_{1}(g) \Phi_{+}, g \in \widetilde{\mathcal{P}_{+}^{\uparrow}}$, is also a straightforward calculation if one recalls that

$$
e^{\frac{i}{2} \theta(A, p)}=\left(\begin{array}{ll}
0 & 0 \\
0 & 1
\end{array}\right) \overline{H_{p}^{-1} A H_{q}}\left(\begin{array}{ll}
0 & 0 \\
0 & 1
\end{array}\right) \quad \text { and that } D^{\left(0, \frac{n}{2}\right)}\left(H_{p}\left(\begin{array}{ll}
1 & 0 \\
0 & 0
\end{array}\right)\right) \varphi(p) \in \mathfrak{H}_{n,+}^{(0)},
$$

for any suitable $\mathcal{H}$-valued function $\varphi$.

3.4 Remark The representations $V_{1,2}$ on the spaces $\mathfrak{H}_{n, \pm}$ are the analogue of the massive representations that avoid the use of so-called 'Wigner rotations' (see e.g. 44, 49] or [9, Part A]). The price for the masslessness here are the degenerate subspaces $\mathfrak{H}_{n, \pm}^{(0)}$. The advantage of using these spaces is the fact that one can more naturally reduce the covariant representation (11) in terms of $V_{1,2}^{\prime}$ than in terms of $U_{ \pm}$given in eq. (17). This reduction is done by means of the embedding $\mathfrak{I}$, which is the essential essential object for the construction of the free net (see also [41]).

Recall next the covariant and massless canonical representations (the latter written in the more convenient factor space notation) considered before:

$$
\begin{gathered}
(T(g) f)(x):=D^{\left(0, \frac{n}{2}\right)}(A) f\left(\Lambda_{A}^{-1}(x-a)\right), \quad g=(A, a) \in \widetilde{\mathcal{P}_{+}^{\uparrow}}, f \in \mathcal{S}\left(\mathbb{R}^{4}, \mathcal{H}^{\left(0, \frac{n}{2}\right)}\right), \\
\left(V_{1}^{\prime}(g)[\varphi]_{+}\right)(p):=\left[e^{-i p a} D^{\left(0, \frac{n}{2}\right)}(A) \varphi\left(\Lambda_{A}^{-1} p\right)\right]_{+},\left(V_{3}^{\prime}(g)[\varphi]_{+}\right)(p):=\left[e^{i p a} D^{\left(0, \frac{n}{2}\right)}(A) \varphi\left(\Lambda_{A}^{-1} p\right)\right]_{+}, \\
\left(V_{2}^{\prime}(g)[\psi]_{-}\right)(p):=\left[e^{-i p a} D^{\left(\frac{n}{2}, 0\right)}(A) \psi\left(\Lambda_{A}^{-1} p\right)\right]_{-},\left(V_{4}^{\prime}(g)[\psi]_{-}\right)(p):=\left[e^{i p a} D^{\left(\frac{n}{2}, 0\right)}(A) \psi\left(\Lambda_{A}^{-1} p\right)\right]_{-},
\end{gathered}
$$

where $\varphi \in \mathfrak{H}_{n,+}$ and $\psi \in \mathfrak{H}_{n,-} . V_{1}^{\prime}$ and $V_{2}^{\prime}$ satisfy the spectrality condition.

The following definition will be the essential ingredient for the massless free net construction:

3.5 Definition Reference spaces and embeddings for the bosonic and fermionic cases: 
(i) In the Bose case ( $n$ even) take $h_{n}:=\mathfrak{H}_{n,+}^{\prime} \oplus \mathfrak{H}_{n,-}^{\prime}$ (considered as a real space) and the symplectic form $\sigma_{n}:=\operatorname{Im}\langle\cdot, \cdot\rangle_{\beta_{+}} \oplus \operatorname{Im}\langle\cdot, \cdot\rangle_{\beta_{-}}$. As symplectic representation of $\widetilde{\mathcal{P}_{+}^{\uparrow}}$ choose $V_{n}:=V_{1}^{\prime} \oplus V_{2}^{\prime}$. The embedding $\mathfrak{I}_{n}: \mathcal{S}\left(\mathbb{R}^{4}, \mathcal{H}^{\left(0, \frac{n}{2}\right)}\right) \rightarrow h_{n}$ is given here by

$$
\left(\mathfrak{I}_{n} f\right)(p):=[\widehat{f}(p)]_{+} \oplus\left[\widehat{\Gamma_{0} f}(p)\right]_{-}, p \in \mathcal{C}_{+},
$$

where $\Gamma_{0}: \mathcal{H}^{\left(0, \frac{n}{2}\right)} \rightarrow \mathcal{H}^{\left(\frac{n}{2}, 0\right)}$ is an antiunitary involution and $\widehat{f}(p):=\int_{\mathbb{R}^{4}} e^{-i p x} f(x) \mathrm{d}^{4} x$ is the Fourier transform.

(ii) In the Fermi case $\left(n\right.$ odd) take $h_{n}:=\mathfrak{H}_{n,+}^{\prime} \oplus \mathfrak{H}_{n,-}^{\prime} \oplus \mathfrak{H}_{n,+}^{\prime} \oplus \mathfrak{H}_{n,-}^{\prime}$ with the natural scalar product and the antilinear involution given by

$$
\Gamma_{n}\left(\left[\varphi_{+}\right]_{+} \oplus\left[\varphi_{-}\right]_{-} \oplus\left[\psi_{+}\right]_{+} \oplus\left[\psi_{-}\right]_{-}\right):=\left[\Gamma_{0} \psi_{-}\right]_{+} \oplus\left[\Gamma_{0} \psi_{+}\right]_{-} \oplus\left[\Gamma_{0} \varphi_{-}\right]_{+} \oplus\left[\Gamma_{0} \varphi_{+}\right]_{-}
$$

As unitary representation of $\widetilde{\mathcal{P}_{+}^{\uparrow}}$ that intertwines with $\Gamma_{n}$ choose $V_{n}:=V_{1}^{\prime} \oplus V_{2}^{\prime} \oplus V_{3}^{\prime} \oplus V_{4}^{\prime}$. In the present case the embedding $\mathfrak{I}_{n}: \mathcal{S}\left(\mathbb{R}^{4}, \mathcal{H}^{\left(0, \frac{n}{2}\right)}\right) \rightarrow h_{n}$ is given by

$$
\left(\Im_{n} f\right)(p):=[\widehat{f}(p)]_{+} \oplus\left[\widehat{\Gamma_{0} f}(p)\right]_{-} \oplus[\widehat{f}(-p)]_{+} \oplus\left[\widehat{\Gamma_{0} f}(-p)\right]_{-}, p \in \mathcal{C}_{+}
$$

The preceding embeddings characterize in a canonical way nets of $\mathrm{C}^{*}$-subalgebras of the CAR- and CCR-algebras associated to the corresponding reference space $h_{n}$ (cf. [10, Chapter 8] and references cited therein). The explicit construction of the net and the verification of some of the main axioms of algebraic QFT is the content of the following theorem.

3.6 Theorem Denoting by $\mathcal{B}\left(\mathbb{R}^{4}\right)$ the set of open and bounded regions in Minkowski space we have

(i) Fermionic case $(n$ odd):

$$
\mathcal{B}\left(\mathbb{R}^{4}\right) \ni \mathcal{O} \longmapsto \mathcal{A}_{n}(\mathcal{O}):=\mathrm{C}^{*}\left(\left\{A\left(\mathfrak{I}_{n} f\right) \mid \operatorname{supp} f \subset \mathcal{O}\right\}\right)^{\mathbb{Z}_{2}} \subset \operatorname{CAR}\left(h_{n}, \Gamma_{n}\right) .
$$

Here $A(\cdot)$ are the generators of the $C A R$-algebra $\operatorname{CAR}\left(h_{n}, \Gamma_{n}\right)$ and $\mathcal{A}^{\mathbb{Z}_{2}}$ denotes the fixed point subalgebra of the $C^{*}$-algebra $\mathcal{A}$ w.r.t. Bogoljubov automorphism associated to the unitarity $-\mathbb{1 1}$.

(ii) Bosonic case ( $n$ even):

$$
\mathcal{B}\left(\mathbb{R}^{4}\right) \ni \mathcal{O} \longmapsto \mathcal{A}_{n}(\mathcal{O}):=\mathrm{C}^{*}\left(\left\{\delta_{\mathfrak{I}_{n} f} \mid \operatorname{supp} f \subset \mathcal{O}\right\}\right) \subset \operatorname{CCR}\left(h_{n}, \sigma_{n}\right)
$$

Here $\delta_{(\cdot)}$ denote the Weyl elements that generate the CCR-algebra $\operatorname{CCR}\left(h_{n}, \sigma_{n}\right)$. 
Finally, the net $\mathcal{B}\left(\mathbb{R}^{4}\right) \ni \mathcal{O} \mapsto \mathcal{A}_{n}(\mathcal{O})$ characterized by the corresponding embeddings $\mathfrak{I}_{n}, n \in \mathbb{N}$, satisfies the properties of

(i) (Isotony) If $\mathcal{O}_{1} \subseteq \mathcal{O}_{2}$, then $\mathcal{A}_{n}\left(\mathcal{O}_{1}\right) \subseteq \mathcal{A}_{n}\left(\mathcal{O}_{2}\right), \mathcal{O}_{1}, \mathcal{O}_{2} \in \mathcal{B}\left(\mathbb{R}^{4}\right)$.

(ii) (Causality) If $\mathcal{O}_{1}$ and $\mathcal{O}_{2}$ are causaly separated, then $\left[\mathcal{A}_{n}\left(\mathcal{O}_{1}\right), \mathcal{A}_{n}\left(\mathcal{O}_{2}\right)\right]=0$.

(iii) (Additivity) For any $\left\{\mathcal{O}_{\lambda}\right\}_{\lambda \in \Lambda} \subset \mathcal{B}\left(\mathbb{R}^{4}\right)$ with $\cup_{\lambda} \mathcal{O}_{\lambda} \in \mathcal{B}\left(\mathbb{R}^{4}\right)$. Then

$$
\mathcal{A}_{n}\left(\cup_{\lambda} \mathcal{O}_{\lambda}\right)=\mathrm{C}^{*}\left(\cup_{\lambda} \mathcal{A}_{n}\left(\mathcal{O}_{\lambda}\right)\right)
$$

(iv) (Covariance) There exists a representation $\widetilde{\mathcal{P}_{+}^{\uparrow}} \ni g \mapsto \alpha_{g}$ in terms of automorphisms of the $C A R$-resp. CCR-algebras such that $\alpha_{g}\left(\mathcal{A}_{n}(\mathcal{O})\right)=\mathcal{A}(g \mathcal{O}), g \in \widetilde{\mathcal{P}_{+}^{\uparrow}}, \mathcal{O} \in \mathcal{B}\left(\mathbb{R}^{4}\right)$.

Proof: Since in this paper the covariance axiom plays a distinguished role we will show only this property here. For the other properties and further details we refer to [9, 43, 44]. We will show that the covariance relation is based on the following intertwining property of the embeddings $\mathfrak{I}_{n}$ w.r.t. the covariant and the canonical representations:

$$
\mathfrak{I}_{n} T(g)=V_{n}(g) \mathfrak{I}_{n}, \quad g \in \widetilde{\mathcal{P}_{+}^{\uparrow}} .
$$

Indeed, let $\alpha_{g}$ be the Bogoljubov automorphisms associated to the Bogoljubov unitaries $V_{n}(g)$. Further note also that the covariant representation $T$ shifts the space time regions in the correct way, i.e. if $f \in \mathcal{S}\left(\mathbb{R}^{4}, \mathcal{H}\right)$ and $\operatorname{supp} f \subset \mathcal{O}$, then $\operatorname{supp}(T(g) f) \subset g \mathcal{O}, g \in \widetilde{\mathcal{P}_{+}^{\uparrow}}$. Now in the bosonic case $\left(n\right.$ even) we have for any $\mathcal{O} \in \mathcal{B}\left(\mathbb{R}^{4}\right), g \in \widetilde{\mathcal{P}_{+}^{\uparrow}}$,

$$
\begin{aligned}
\alpha_{g}(\mathcal{A}(\mathcal{O})) & =\alpha_{g}\left(\mathrm{C}^{*}\left(\left\{\delta_{\mathfrak{I}_{n} f} \mid \operatorname{supp} f \subset \mathcal{O}\right\}\right)\right) \\
& =\mathrm{C}^{*}\left(\left\{\alpha_{g}\left(\delta_{\mathfrak{I}_{n} f}\right) \mid \operatorname{supp} f \subset \mathcal{O}\right\}\right) \\
& =\mathrm{C}^{*}\left(\left\{\delta_{V_{n}(g)\left(\mathfrak{I}_{n} f\right)} \mid \operatorname{supp} f \subset \mathcal{O}\right\}\right) \\
& =\mathrm{C}^{*}\left(\left\{\delta_{\mathfrak{I}_{n}(T(g) f)} \mid \operatorname{supp} f \subset \mathcal{O}\right\}\right) \\
& =\mathrm{C}^{*}\left(\left\{\delta_{\mathfrak{I}_{n} f^{\prime}} \mid \operatorname{supp} f^{\prime} \subset g \mathcal{O}\right\}\right) \\
& =\mathcal{A}(g \mathcal{O}) .
\end{aligned}
$$

One can argue similarly for the fermionic nets.

3.7 Remark Note that for the free nets constructed previously the one particle Hilbert space corresponding to the canonical Fock states (to be specified in Section 5 ) is $\mathfrak{H}_{n,+}^{\prime} \oplus \mathfrak{H}_{n,-}^{\prime}, n \in \mathbb{N}$. It carries a representation $V_{1}^{\prime} \oplus V_{2}^{\prime}$ which by Theorem 3.3 is equivalent to the reducible Wigner 
massless representations $U_{+} \oplus U_{-}$with helicities $\frac{n}{2}$ and $-\frac{n}{2}$. This Hilbert space and representation coincide with the one-particle Hilbert space used in more standard quantum field theoretical construction of massless free fields (cf. e.g. 31]).

We will show later that the covariance property of the massless free nets can be extended to the fourfold coverning of the conformal group $\mathrm{SU}(2,2)$.

\section{Extension of the massless representations}

The first step to show that the massless free nets contructed in the previous theorem are also covariant w.r.t. the conformal group is to show that the massless canonical representations $V_{k}^{\prime}$, $k=1,2,3,4$, extend within $\mathfrak{H}_{ \pm}^{\prime}$ to a unitary representation of $\mathrm{SU}(2,2)$. This fact has been shown considering different mathematical contexts (see e.g. 45, 18, 33, 2]). We will give next a simplified proof of this result due to the nice properties the functions $\beta_{ \pm}(\cdot)$ introduced in the previous section.

In the context of Subsection 2.2 we consider as inducing representation of the little group $\mathcal{K}_{0}$ on $\mathcal{H}^{\left(0, \frac{n}{2}\right)}$

$$
\tau(K):=\operatorname{det}(A-i B) D^{\left(0, \frac{n}{2}\right)}(A-i B), \quad K=\left(\begin{array}{cc}
A & B \\
-B & A
\end{array}\right) \in \mathcal{K}_{0},
$$

which is unitary because from Eqs. (19) we have in this case $A A^{*}+B B^{*}=11, A B^{*}=-B A^{*}$ etc. The first step will be to define a mapping $J$ that satisfies the properties required in Remark 2.1 (ii).

4.1 Lemma For $g=\left(\begin{array}{cc}A_{1} & A_{2} \\ A_{3} & A_{4}\end{array}\right) \in \mathrm{SU}(2,2), Z \in \mathbb{T}_{+}$, the mapping

$$
J^{\left(0, \frac{n}{2}\right)}(g, Z):=\operatorname{det}\left(A_{3} Z+A_{4}\right) D^{\left(0, \frac{n}{2}\right)}\left(A_{3} Z+A_{4}\right)
$$

satisfies the properties

$$
\begin{aligned}
J^{\left(0, \frac{n}{2}\right)}\left(g_{1} g_{2}, Z\right) & =J^{\left(0, \frac{n}{2}\right)}\left(g_{1}, g_{2} Z\right) J^{\left(0, \frac{n}{2}\right)}\left(g_{2}, Z\right), \quad g_{1}, g_{2} \in \mathrm{SU}(2,2), Z \in \mathbb{T}_{+}, \\
J^{\left(0, \frac{n}{2}\right)}(\mathbb{1}, Z) & =\mathbb{1} \\
J^{\left(0, \frac{n}{2}\right)}(K, i 1) & =\tau(K), \quad K \in \mathcal{K}_{0} .
\end{aligned}
$$

Proof: The second property is trivial since $J^{\left(0, \frac{n}{2}\right)}(\mathbb{1}, Z)=\operatorname{det}(\mathbb{1}) D^{\left(0, \frac{n}{2}\right)}(\mathbb{1})=\mathbb{1}_{\mathcal{H}}$. Further, the third eq. follows also directly from the choices of $\tau$ and $J$. To prove the first property put $J_{1}(g, Z):=A_{3} Z+A_{4}$ (which acts on $\mathbb{C}^{2}$ ) and note that it already satisfies the condition $J_{1}\left(g_{1} g_{2}, Z\right)=J_{1}\left(g_{1}, g_{2} Z\right) J_{1}\left(g_{2}, Z\right)$ as can be immediately checked. Therefore, since 
$D(\cdot)$ is a representation and from the product rule for determinants it follows that $J(g, Z)=$ $\operatorname{det}\left(J_{1}(g, Z)\right) D\left(J_{1}(g, Z)\right)$ satisfies the required condition.

4.2 Remark The following results will be close to those in [33, Section IV]. The main difference w.r.t. Jakobsen and Vergne's approach lies in the fact the we are working with Minkowski scalar products in the arguments of the exponentials that appear, while in the cited reference mainly euclidean scalar products are considered. This variation will have no consequence for the absolute convergence of the integrals studied next and it will considerably simplify some proofs later on, e.g. the extension result for the massless canonical representation of the Poincaré group (cf. Theorem 4.6). Note that we can write $x p=x_{0} p_{0}-\sum_{i} x_{i} p_{i}=\operatorname{Tr}\left(P^{\dagger} X\right)$ and $x_{0} p_{0}+\sum_{i} x_{i} p_{i}=$ $\frac{1}{2} \operatorname{Tr}(P X)$, where $P, P^{\dagger}$ are given in Section 3. The following two technical lemmas will be essential for the proof of the conformal covariance of massless free nets.

4.3 Lemma For $Y \in \mathrm{H}_{+}(2, \mathbb{C})$ we have

$$
\int_{\mathcal{C}_{+}} e^{-\operatorname{Tr}\left(P^{\dagger} Y\right)} \beta_{+}(p) \mu_{0}(\mathrm{~d} p)=C_{n}(\operatorname{det} Y)^{-1} D^{\left(0, \frac{n}{2}\right)}(Y)^{-1}, \quad C_{n}>0,
$$

where the l.h.s. is an absolutely convergent integral.

Proof: First note that with the notation above $\overline{P^{\dagger}}=\frac{1}{2}\left(\begin{array}{cc}0 & -1 \\ 1 & 0\end{array}\right) P\left(\begin{array}{cc}0 & 1 \\ -1 & 0\end{array}\right)$ so that

$$
\begin{aligned}
& \int_{\mathcal{C}_{+}} e^{-\operatorname{Tr}\left(P^{\dagger} Y\right)} \beta_{+}(p) \mu_{0}(\mathrm{~d} p) \\
&=\left(\frac{1}{2}\right)^{n} D^{\left(0, \frac{n}{2}\right)}\left(\left(\begin{array}{cc}
0 & -1 \\
1 & 0
\end{array}\right)\right) \\
&\left(\int_{\mathcal{C}_{+}} e^{-\operatorname{Tr}\left(P\left(\begin{array}{cc}
0 & 1 \\
-1 & 0
\end{array}\right)\left(\frac{1}{2} Y^{t}\right)\left(\begin{array}{cc}
0 & -1 \\
1 & 0
\end{array}\right)\right)} D^{\left(\frac{n}{2}, 0\right)}(P) \mu_{0}(\mathrm{~d} p)\right) D^{\left(0, \frac{n}{2}\right)}\left(\left(\begin{array}{cc}
0 & 1 \\
-1 & 0
\end{array}\right)\right),
\end{aligned}
$$

where the index $t$ means matrix transposition. But from [33, Proposition IV.1.1] the integral on the r.h.s. of the preceding equation is absolutely convergent for $Y \in \mathrm{H}_{+}(2, \mathbb{C})$ and even more we also have from the mentioned proposition that for some $C_{n}^{\prime}>0$

$$
\int_{\mathcal{C}_{+}} e^{-\operatorname{Tr}\left(P\left(\begin{array}{cc}
0 & 1 \\
-1 & 0
\end{array}\right)\left(\frac{1}{2} Y^{t}\right)\left(\begin{array}{cc}
0 & -1 \\
1 & 0
\end{array}\right)\right)} D^{\left(\frac{n}{2}, 0\right)}(P) \mu_{0}(\mathrm{~d} p)=C_{n}^{\prime}(\operatorname{det} Y)^{-1} D^{\left(\frac{n}{2}, 0\right)}\left(\frac{1}{2}\left(\begin{array}{cc}
0 & 1 \\
-1 & 0
\end{array}\right) Y^{t}\left(\begin{array}{cc}
0 & -1 \\
1 & 0
\end{array}\right)\right)^{-1} .
$$

Inserting this result on the r.h.s. of Eq. (33) and using $D^{\left(\frac{n}{2}, 0\right)}\left(Y^{t}\right)=D^{\left(0, \frac{n}{2}\right)}(Y)$ we get the eq. of the lemma. 
4.4 Lemma For $Z_{1}, Z_{2} \in \mathbb{T}_{+}$we have

$$
\mathfrak{K}_{+}\left(Z_{1}, Z_{2}\right):=C_{n}\left(\operatorname{det}\left(\frac{Z_{1}-Z_{2}^{*}}{2 i}\right)\right)^{-1} D^{\left(0, \frac{n}{2}\right)}\left(\frac{Z_{1}-Z_{2}^{*}}{2 i}\right)^{-1}=\int_{\mathcal{C}_{+}} e^{i \operatorname{Tr}\left(P^{\dagger}\left(Z_{1}-Z_{2}^{*}\right)\right)} \beta_{+}(p) \mu_{0}(\mathrm{~d} p),
$$

where the integral is absolutely convergent. Further, for any $g \in \mathrm{SU}(2,2)$ we have

$$
\mathfrak{K}_{+}\left(g Z_{1}, g Z_{2}\right)=J^{\left(0, \frac{n}{2}\right)}\left(g, Z_{1}\right) \mathfrak{K}_{+}\left(Z_{1}, Z_{2}\right)\left(J^{\left(0, \frac{n}{2}\right)}\left(g, Z_{2}\right)\right)^{*}
$$

Proof: Note first that if $Z_{1}, Z_{2} \in \mathbb{T}_{+}$, then $Z_{1}-Z_{2}^{*} \in \mathbb{T}_{+}$, which $\operatorname{implies} \operatorname{det}\left(Z_{1}-Z_{2}^{*}\right) \neq 0$. Applying now Lemma 4.3 as well as [33, Proposition IV.1.2] we get the first part of the statement. To prove the last equation take $g=\left(\begin{array}{cc}A_{1} & A_{2} \\ A_{3} & A_{4}\end{array}\right) \in \mathrm{SU}(2,2)$ and consider first

$$
\begin{aligned}
\frac{1}{2 i}\left(g Z_{1}-\left(g Z_{2}\right)^{*}\right)^{-1}= & \frac{1}{2 i}\left(\left(A_{1} Z_{1}+A_{2}\right)\left(A_{3} Z_{1}+A_{4}\right)^{-1}-\left(\left(A_{1} Z_{2}+A_{2}\right)\left(A_{3} Z_{2}+A_{4}\right)^{-1}\right)^{*}\right)^{-1} \\
= & \frac{1}{2 i}\left(A_{3} Z_{1}+A_{4}\right) \cdot \\
& \left(\left(Z_{2}^{*} A_{3}^{*}+A_{4}^{*}\right)\left(A_{1} Z_{1}+A_{2}\right)-\left(Z_{2}^{*} A_{1}^{*}+A_{2}^{*}\right)\left(A_{3} Z_{1}+A_{4}\right)\right)^{-1} \cdot\left(A_{3} Z_{2}+A_{4}\right)^{*} \\
= & \left(A_{3} Z_{1}+A_{4}\right) \cdot \frac{1}{2 i}\left(Z_{1}-Z_{2}^{*}\right)^{-1} \cdot\left(A_{3} Z_{2}+A_{4}\right)^{*},
\end{aligned}
$$

where for the last eq. we have used the relations (19). Now recalling the definition of $J$ in Lemma 4.1 we have that

$$
\begin{aligned}
\mathfrak{K}_{+}\left(g Z_{1}, g Z_{2}\right) & =C_{n}\left(\operatorname{det}\left(\frac{g Z_{1}-\left(g Z_{2}\right)^{*}}{2 i}\right)\right)^{-1} D^{\left(0, \frac{n}{2}\right)}\left(\frac{g Z_{1}-\left(g Z_{2}\right)^{*}}{2 i}\right)^{-1} \\
& =J^{\left(0, \frac{n}{2}\right)}\left(g, Z_{1}\right) \mathfrak{K}_{+}\left(Z_{1}, Z_{2}\right)\left(J^{\left(0, \frac{n}{2}\right)}\left(g, Z_{2}\right)\right)^{*}
\end{aligned}
$$

and the proof is concluded.

We will explicitly give next a parametrization in terms of the sets $\mathbb{T}_{+}$and $\mathcal{H}$ of a total set $\mathfrak{H}_{\text {tot }}^{\prime} \subset \mathfrak{H}_{n,+}^{\prime}$ that will satisfy the properties required in Subsection 2.2.

4.5 Lemma The set $\mathfrak{H}_{\text {tot }}^{\prime}:=\left\{\left[K_{Z, v}\right]_{+} \mid K_{Z, v}(p):=e^{-i \operatorname{Tr}\left(P^{\dagger} Z^{*}\right)} v, p \in \mathcal{C}_{+}, Z \in \mathbb{T}_{+}, v \in \mathcal{H}\right\}$ is total in $\mathfrak{H}_{n,+}^{\prime}$. Further the following equation holds for all $g \in \mathrm{SU}(2,2)$ :

$$
\left\langle K_{g Z_{1}, v_{1}}, K_{g Z_{2}, v_{2}}\right\rangle_{\beta_{+}}=\left\langle K_{Z_{1}, J\left(g, Z_{1}\right)^{*} v_{1}}, K_{Z_{2}, J\left(g, Z_{2}\right)^{*} v_{2}}\right\rangle_{\beta_{+}}, \quad Z_{1}, Z_{2} \in \mathbb{T}_{+}, v_{1}, v_{2} \in \mathcal{H}
$$

Proof: First from Lemma 4.3 we have for any $Z=X+i Y \in \mathbb{T}_{+}, v \in \mathcal{H}$, that

$$
\left\langle K_{Z, v}, K_{Z, v}\right\rangle_{\beta_{+}}=\int_{\mathcal{C}_{+}} e^{-\operatorname{Tr}\left(2 P^{\dagger} Y\right)}\left\langle v, \beta_{+}(p) v\right\rangle_{\mathcal{H}} \mu_{0}(\mathrm{~d} p)<\infty
$$


and span $\mathfrak{H}_{\text {tot }}^{\prime}$ is dense in $\mathfrak{H}_{n,+}^{\prime}$ by Lemmas 4.2 .2 and 4.2.3 in 18 .

Finally, recalling the properties of $\mathfrak{K}_{+}$in Lemma 4.4 we have

$$
\begin{aligned}
\left\langle K_{g Z_{1}, v_{1}}, K_{g Z_{2}, v_{2}}\right\rangle_{\beta_{+}} & =\int_{\mathcal{C}_{+}}\left\langle e^{-i \operatorname{Tr}\left(P^{\dagger}\left(g Z_{1}\right)^{*}\right)} v_{1}, \beta_{+}(p) e^{-i \operatorname{Tr}\left(P^{\dagger}\left(g Z_{2}\right)^{*}\right)} v_{2}\right\rangle_{\mathcal{H}} \mu_{0}(\mathrm{~d} p) \\
& =\langle v_{1}, \underbrace{\left(\int_{\mathcal{C}_{+}} e^{i \operatorname{Tr}\left(P^{\dagger}\left(g Z_{1}-\left(g Z_{2}\right)^{*}\right)\right.} \beta_{+}(p) \mu_{0}(\mathrm{~d} p)\right)}_{\mathfrak{K}_{+}\left(g Z_{1}, g Z_{2}\right)} v_{2}\rangle_{\mathcal{H}} \\
& =\int_{\mathcal{C}_{+}}\left\langle e^{-i \operatorname{Tr}\left(P^{\dagger} Z_{1}^{*}\right)} J\left(g, Z_{1}\right)^{*} v_{1}, \beta_{+}(p) e^{-i \operatorname{Tr}\left(P^{\dagger} Z_{2}^{*}\right)} J\left(g, Z_{2}\right)^{*} v_{2}\right\rangle_{\mathcal{H}} \mu_{0}(\mathrm{~d} p) \\
& =\left\langle K_{Z_{1}, J\left(g, Z_{1}\right)^{*} v_{1}}, K_{Z_{2}, J\left(g, Z_{2}\right)^{*} v_{2}}\right\rangle_{\beta_{+}},
\end{aligned}
$$

and the proof is concluded.

4.6 Theorem The following representation of the conformal group defined on $\mathfrak{H}_{\text {tot }}^{\prime}$ by

$$
W_{1}^{\prime}(g)\left[K_{Z, v}\right]_{+}(p):=\left[K_{g Z,\left(J(g, Z)^{-1}\right)^{*} v}\right]_{+}(p), \quad g \in \mathrm{SU}(2,2)
$$

extends to a unitary and irreducible representation within $\mathfrak{H}_{n,+}^{\prime} \cdot$ Further, the restriction of $W_{1}^{\prime}$ to the Poincaré subgroup coincides with $V_{1}^{\prime}$ defined in Section 3 , which is equivalent to the massless canonical representation of helicity $\frac{n}{2}$.

Proof: First of all note that by the proof of Lemma 4.5 we have that $K_{Z, v} \mapsto K_{g Z,\left(J(g, Z)^{-1}\right)^{*} v}$ leaves the sesquilinear form $\langle\cdot, \cdot\rangle_{\beta_{+}}$invariant and therefore the definition of $W_{1}^{\prime}$ on the factor space is consistent with the corresponding equivalence classes. Now again by Lemma 4.5 we can apply Lemma 2.3 to the present situation to conclude that $W_{1}^{\prime}$ extends to a unitary representation within $\mathfrak{H}_{n,+}^{\prime}$.

Consider next the Poincaré subgroup of $\mathrm{SU}(2,2)$ given in Eq. (21), i.e.

$$
\mathrm{SU}(2,2) \supset \widetilde{\mathcal{P}_{+}^{\uparrow}} \ni g_{0}=\left(\begin{array}{cc}
A & C\left(A^{*}\right)^{-1} \\
0 & \left(A^{*}\right)^{-1}
\end{array}\right), A \in \mathrm{SL}(2, \mathbb{C}), C=C^{*} \in \mathrm{H}(2, \mathbb{C}) .
$$

For this subgroup and recalling the action in Eq. (20) as well as Remark 4.2 we have

$$
e^{-i \operatorname{Tr}\left(P^{\dagger}\left(g_{0} Z\right)^{*}\right)}=e^{-i \operatorname{Tr}\left(P^{\dagger} C\right)} e^{-i \operatorname{Tr}\left(P^{\dagger} A Z^{*} A^{*}\right)}=e^{-i \operatorname{Tr}\left(P^{\dagger} C\right)} e^{-i \operatorname{Tr}\left(\left(A^{-1} P\left(A^{-1}\right)^{*}\right)^{\dagger} Z^{*}\right)}
$$

and also $J^{\left(0, \frac{n}{2}\right)}\left(g_{0}, Z\right)=D^{\left(0, \frac{n}{2}\right)}\left(A^{-1}\right)^{*}$. From this we get

$$
W_{1}^{\prime}\left(g_{0}\right)\left[K_{Z, v}\right]_{+}(p)=\left[e^{-i \operatorname{Tr}\left(P^{\dagger} C\right)} D^{\left(0, \frac{n}{2}\right)}(A) K_{Z, v}\left(\Lambda_{A}^{-1} p\right)\right]_{+} .
$$


Thus the unitary representations $W_{1}^{\prime} \widetilde{\mathcal{P}_{+}^{\uparrow}}$ and $V_{1}^{\prime}$ coincide on a total set and therefore they must be equal. Now, $V_{1}^{\prime}$ is equivalent to massless canonical representation with helicity $\frac{n}{2}$ and since $V_{1}^{\prime}=W_{1}^{\prime} \widetilde{\mathcal{P}_{+}^{\uparrow}}$ is already irreducible, then $W_{1}^{\prime}$ is certainly irreducible for the whole $\operatorname{SU}(2,2)$.

4.7 Remark (i) Note that the representation $W_{1}^{\prime}$ is just the transcription of the induced representation considered Remark 2.1 (ii) in terms of the more useful set of functions $\mathfrak{H}_{\text {tot }}^{\prime}$. Indeed, recalling the kernels introduced in Lemma 4.4 consider the following functions $\varphi_{Z_{0}, v}: \mathbb{T}_{+} \rightarrow \mathcal{H}, Z_{0} \in \mathbb{T}_{+}, v \in \mathcal{H}$

$$
\varphi_{Z_{0}, v}(Z):=\mathfrak{K}_{+}\left(Z_{0}, Z\right) v=\int_{\mathcal{C}_{+}} e^{i \operatorname{Tr}\left(P^{\dagger} Z_{0}\right)} \beta_{+}(p) K_{Z, v}(p) \mu_{0}(\mathrm{~d} p) .
$$

Using again Lemma 4.4 it is now straightforward to rewrite the induced representation (9) for the functions $\varphi_{Z_{0}, v}$ in terms of the functions $K_{Z, v}$.

(ii) We can argue similarly as in this section for the spaces with opposite helicity. Indeed, use the mapping $J^{\left(\frac{n}{2}, 0\right)}(g, Z):=\Gamma_{0} J^{\left(0, \frac{n}{2}\right)}(g, Z) \Gamma_{0}$ and the kernel $\mathfrak{K}_{-}\left(Z_{1}, Z_{2}\right)=\Gamma_{0} \mathfrak{K}_{+}\left(Z_{1}, Z_{2}\right) \Gamma_{0}$. It can be easily seen now that we can extend as in the preceding theorem the representations $V_{i}^{\prime}$ needed in the previous section to the define the free nets to corresponding representations $W_{i}^{\prime}, i=2,3,4$.

\section{Conformal covariance and its consequences}

One of the characteristic facts about the conformal group is that it acts quasi-globaly on Minkowski space. This behaviour is due to the fact that the subgroup of the special conformal transformations has always singularities on certain hypersurfaces of $\mathbb{R}^{4}$. We will therefore restrict in this section to $g \in \mathrm{SU}(2,2), f \in \mathrm{C}_{0}^{\infty}\left(\mathbb{R}^{4}, \mathcal{H}\right)$ and double cones $\mathcal{O} \in \mathcal{B}\left(\mathbb{R}^{4}\right)$, where $g \operatorname{supp} f$ and $g \mathcal{O} \subset \mathbb{R}^{4}$ are well defined. We denote the family of double cones in $\mathbb{R}^{4}$ by $\mathcal{K}$. These are standard assumptions in order to understand the axiom of covariance in the general setting of conformal quantum field theory (cf. [13, Section 1], [55, I.4]).

To apply next the explicit formulas concerning the representations of $\mathrm{SU}(2,2)$ considered in the preceding section, we will need to introduce first a suitable $y$-dependent embedding $\left(y \in \mathcal{V}_{+}\right.$, i.e. $\left.Y \in \mathrm{H}_{+}(2, \mathbb{C})\right)$ which can be related to the embedding needed in Section 3 to the define the free net. 
5.1 Definition Putting $Z=X+i Y \in \mathbb{T}_{+}$we define $\mathfrak{I}_{y,+}: \mathrm{C}_{0}^{\infty}\left(\mathbb{R}^{4}, \mathcal{H}^{\left(0, \frac{n}{2}\right)}\right) \rightarrow \mathfrak{H}_{n,+}^{\prime}$ by

$$
\left(\mathfrak{I}_{y,+} f\right)(p):=\left[\int_{\mathbb{R}^{4}} e^{-i \operatorname{Tr}\left(P^{\dagger} Z^{*}\right)} f(x) \mathrm{d}^{4} x\right]_{+}=\left[\int_{\mathbb{R}^{4}} e^{-i \operatorname{Tr}\left(P^{\dagger}(X-i Y)\right)} f(x) \mathrm{d}^{4} x\right]_{+} .
$$

5.2 Lemma The $y$-dependent embedding satisfies $\mathfrak{I}_{y,+} f \in \mathfrak{H}_{n,+}^{\prime}$ and $\lim _{\mathcal{V}_{+} \ni y \rightarrow 0} \mathfrak{I}_{y,+} f=[\widehat{f}]_{+}$, where the limit exists in the Hilbert space norm $\|\cdot\|_{\beta_{+}}$.

Proof: For any $f \in \mathrm{C}_{0}^{\infty}\left(\mathbb{R}^{4}, \mathcal{H}^{\left(0, \frac{n}{2}\right)}\right)$ it follows from Lemma 4.3 that $\mathfrak{I}_{y,+} f \in \mathfrak{H}_{n,+}^{\prime}$. Further

$$
\begin{aligned}
\left\|\Im_{y,+} f-[\widehat{f}]_{+}\right\|_{\beta_{+}}^{2} & =\int_{\mathcal{C}_{+}}\left\langle\left(\Im_{y,+} f(p)-\widehat{f}(p)\right), \beta_{+}(p)\left(\Im_{y,+} f(p)-\widehat{f}(p)\right)\right\rangle_{\mathcal{H}} \mu_{0}(\mathrm{~d} p) \\
& =\int_{\mathbb{R}^{4}} \int_{\mathbb{R}^{4}} \int_{\mathcal{C}_{+}}\left|e^{-\operatorname{Tr}\left(P^{\dagger} Y\right)}-1\right|^{2}\left\langle f(x), \beta_{+}(p) f\left(x^{\prime}\right)\right\rangle \mu_{0}(\mathrm{~d} p) \mathrm{d}^{4} x \mathrm{~d}^{4} x^{\prime}
\end{aligned}
$$

and the last expression tends to zero as $\mathcal{V}_{+} \ni y \rightarrow 0$ by Lebesgue's dominated convergence theorem (note that for $y \in \mathcal{V}_{+}$we have $1 \geq\left|e^{-\operatorname{Tr}\left(P^{\dagger} Y\right)}-1\right|^{2} \rightarrow 0$ as $\mathcal{V}_{+} \ni y \rightarrow 0$ ).

Now inspired by Theorem 4.6 we can consider the following representation on the set of embedded test functions.

5.3 Definition For $f \in \mathrm{C}_{0}^{\infty}\left(\mathbb{R}^{4}, \mathcal{H}^{\left(0, \frac{n}{2}\right)}\right), g \in \mathrm{SU}(2,2)$ and $Y \in \mathrm{H}_{+}(2, \mathbb{C})$ we define

$$
\left(W_{1}(g)\left(\mathfrak{J}_{y,+} f\right)\right)(p):=\left[\int_{\mathbb{R}^{4}} e^{-i \operatorname{Tr}\left(P^{\dagger}(g Z)^{*}\right)}\left(J^{\left(0, \frac{n}{2}\right)}(g, Z)^{-1}\right)^{*} f(x) \mathrm{d}^{4} x\right]_{+} .
$$

5.4 Lemma The representation defined before satisfies for $f, k \in \mathrm{C}_{0}^{\infty}\left(\mathbb{R}^{4}, \mathcal{H}^{\left(0, \frac{n}{2}\right)}\right)$

$$
\left\langle W_{1}(g) \mathfrak{I}_{y,+} f, W_{1}(g) \mathfrak{I}_{y,+} k\right\rangle_{\beta_{+}}=\left\langle\mathfrak{I}_{y,+} f, \mathfrak{I}_{y,+} k\right\rangle_{\beta_{+}}, \quad g \in \mathrm{SU}(2,2), Z=X+i Y \in \mathbb{T}_{+}
$$

Further we have $W_{1}(g)\left(\mathfrak{I}_{y,+} f\right)=\mathfrak{I}_{g y,+}\left(T_{y}(g) f\right)$, where

$$
\left(T_{y}(g) f\right)(g x):=\left(J^{\left(0, \frac{n}{2}\right)}(g, Z)^{-1}\right)^{*} f(x)
$$

satisfies the relation $T_{y}\left(g_{1} g_{2}\right)=T_{g_{2} y}\left(g_{1}\right) T_{y}\left(g_{2}\right), g_{1}, g_{2} \in \mathrm{SU}(2,2)$.

Proof: The unitarity property is based on Lemma 4.4 (cf. with the proof of Theorem 4.6). The other relations follow immediately from the definition of $T_{y}$. 
5.5 Theorem Consider for suitable $f \in \mathrm{C}_{0}^{\infty}\left(\mathbb{R}^{4}, \mathcal{H}^{\left(0, \frac{n}{2}\right)}\right)$ and $g \in \mathrm{SU}(2,2)$ the representation

$$
\left(W_{1}(g)[\widehat{f}]_{+}\right)(p):=\lim _{\mathcal{V}_{+} \ni g y \rightarrow 0}\left(W_{1}(g)\left(\Im_{y,+} f\right)\right)(p)=\left[\left(\widehat{T_{0}(g)} f\right)(p)\right]_{+},
$$

where $\left(T_{0}(g) f\right)(g x):=\left(J^{\left(0, \frac{n}{2}\right)}(g, X)^{-1}\right)^{*} f(x)$. Further $W_{1} \widetilde{\mathcal{P}_{+}^{\uparrow}}=V_{1}^{\prime}$ on the set $\left\{[\widehat{f}]_{+} \mid f \in\right.$ $\left.\mathrm{C}_{0}^{\infty}\left(\mathbb{R}^{4}, \mathcal{H}^{\left(0, \frac{n}{2}\right)}\right)\right\}$ and $T_{0}$ is a representation of $\mathrm{SU}(2,2)$ on the test functions that satisfies $\operatorname{supp}\left(T_{0}(g) f\right) \subseteq g \operatorname{supp} f$. Finally, $T_{0} \widetilde{\mathcal{P}_{+}^{\uparrow}}$ coincides with the covariant representation $T$ defined in Subsection 2.1.

Proof: From Definition 5.1, Lemma 5.2 and noting that $J(g, Z)^{-1}=J\left(g^{-1}, g Z\right)$ (use Eqs. (6) and (7)) we have that

$$
\lim _{\mathcal{V}_{+} \ni g y \rightarrow 0}\left[\int_{\mathbb{R}^{4}} e^{-i \operatorname{Tr}\left(P^{\dagger}(g Z)^{*}\right)} J^{\left(0, \frac{n}{2}\right)}\left(g^{-1}, g(X+i Y)\right)^{*} f(x) \mathrm{d}^{4} x\right]_{+}=\left[\left(\widehat{T_{0}} \widehat{(g)} f\right)(p)\right]_{+} .
$$

That $W_{1} \widetilde{\mathcal{P}_{+}^{\uparrow}}=V_{1}^{\prime}$ follows by the same arguments as in the proof of Theorem 1.6 . Note also that for suitable $f$ and $g$ as stated above the test function $T_{0}(g) f$ is smooth and the support properties of $T_{0}$ follow immediately from its definition. Finally, we have for elements $g_{0}=\left(\begin{array}{cc}A C\left(A^{*}\right)^{-1} \\ 0 & \left(A^{*}\right)^{-1}\end{array}\right) \in$ $\widetilde{\mathcal{P}_{+}^{\uparrow}}$ that $J^{\left(0, \frac{n}{2}\right)}\left(g_{0}, Z\right)=D^{\left(0, \frac{n}{2}\right)}\left(A^{-1}\right)^{*}$ and this implies $T_{0} \widetilde{\left\lceil\mathcal{P}_{+}^{\uparrow}\right.}=T$ on the space of test functions, where the covariant representation $T$ is given in Eq. (11).

5.6 Remark Taking into account the comments in Remark 4.7 (ii) we can define similarly the representations $W_{i}, i=2,3,4$, and obtain the corresponding intertwining relations with $T_{0}$.

5.7 Theorem The massless free nets given in Theorem 3.6 are $\mathrm{SU}(2,2)$ covariant.

Proof: Putting in the Bose case $\left(n\right.$ even) $W_{n}:=W_{1} \oplus W_{2}$ and in the Fermi case ( $n$ odd) $W_{n}:=W_{1} \oplus W_{2} \oplus W_{3} \oplus W_{4}$, we get from Theorem 5.5 and the preceding remark that for $n$ even $W_{n}$ leaves the symplectic form $\sigma_{n}$ invariant resp. for $n$ odd $W_{n}$ leaves the corresponding scalar product on $h_{n}$ invariant (cf. Definition 3.5). Further, for suitable $g \in \mathrm{SU}(2,2)$ and $f \in \mathrm{C}_{0}^{\infty}\left(\mathbb{R}^{4}, \mathcal{H}^{\left(0, \frac{n}{2}\right)}\right)$ the respective eq.

$$
W_{n}(g) \mathfrak{I}_{n} f=\mathfrak{I}_{n}\left(T_{0}(g) f\right)
$$

hold. Now the covariance follows by similar arguments as in the proof of Theorem 3.6.

We will now make use of the $\mathrm{SU}(2,2)$ covariance proved in the preceding theorem and which is typical of massless free nets. We will show that the models studied in this paper are examples 
of the conformally covariant nets studied in 13. Thus at the level of the von Neumann algebras we will be able to apply the general results of the mentioned reference. First we need to consider the natural Fock states on $\operatorname{CCR}\left(h_{n}, \sigma_{n}\right)$ resp. $\operatorname{CAR}\left(h_{n}, \Gamma_{n}\right)$. For $n$ even the Fock state is specified by the natural internal complexification of $h_{n}, j\left(\varphi_{+} \oplus \varphi_{-}\right):=i \varphi_{+} \oplus i \varphi_{-}$, while for $n$ odd the Fock state characterized by the basis projection $P:=\left(\begin{array}{llll}1 & 0 & 0 & 0 \\ 0 & 11 & 0 & 0 \\ 0 & 0 & 0 & 0 \\ 0 & 0 & 0 & 0\end{array}\right)$ on $h_{n}$ (cf. [10, Chapter 8], [9]). Note that in both cases the one particle Hilbert space is given by $h_{n}:=\mathfrak{H}_{n,+}^{\prime} \oplus \mathfrak{H}_{n,-}^{\prime}$ and the unitary reducible representation $W_{1}^{\prime} \oplus W_{2}^{\prime}$ satisfy the spectrality condition on it (recall also Remark 3.7). Let $\pi_{0}$ be the Fock representation on the corresponding symmetric resp. antisymmetric Fock space $\mathfrak{H}_{0}$ with Fock vacuum $\Omega$ and denote by a prime the commutant in $\mathcal{B}\left(\mathfrak{H}_{0}\right)$. Then we may consider the following net of von Neumann algebras indexed by double cones:

$$
\mathcal{K} \ni \mathcal{O} \mapsto \mathcal{M}_{n}(\mathcal{O}):=\left(\pi_{0}\left(\mathcal{A}_{n}(\mathcal{O})\right)\right)^{\prime \prime} \subset \mathcal{B}\left(\mathfrak{H}_{0}\right)
$$

We will show next that the preceding net $\mathcal{O} \mapsto \mathcal{M}_{n}(\mathcal{O})$ satisfies the axioms of a vacuum representation (cf. [8, Chapter 1]) with the stronger covariance w.r.t. the conformal group.

5.8 Proposition The nets of von Neumann algebras $\mathcal{O} \mapsto \mathcal{M}_{n}(\mathcal{O}), n \in \mathbb{N}$, defined before satisfy the properties of

(i) (Isotony) If $\mathcal{O}_{1} \subseteq \mathcal{O}_{2}$, then $\mathcal{M}_{n}\left(\mathcal{O}_{1}\right) \subseteq \mathcal{M}_{n}\left(\mathcal{O}_{2}\right), \mathcal{O}_{1}, \mathcal{O}_{2} \in \mathcal{K}$.

(ii) (Causality) If $\mathcal{O}_{1} \perp \mathcal{O}_{2}$, then $\mathcal{M}_{n}\left(\mathcal{O}_{1}\right) \subseteq \mathcal{M}_{n}\left(\mathcal{O}_{2}\right)^{\prime}$.

(iii) (Additivity) For any $\left\{\mathcal{O}_{\lambda}\right\}_{\lambda \in \Lambda} \subset \mathcal{K}$ with $\cup_{\lambda} \mathcal{O}_{\lambda} \in \mathcal{K}$. Then

$$
\mathcal{M}_{n}\left(\cup_{\lambda} \mathcal{O}_{\lambda}\right)=\bigvee_{\lambda} \mathcal{M}_{n}\left(\mathcal{O}_{\lambda}\right):=\left(\cup_{\lambda} \mathcal{M}_{n}\left(\mathcal{O}_{\lambda}\right)\right)^{\prime \prime}
$$

(iv) (Covariance and spectrality condition) There exists a unitary representation $Q$ of $\mathrm{SU}(2,2)$ on $\mathcal{B}\left(\mathfrak{H}_{0}\right)$ and a $Q$-invariant vector $\Omega \in \mathfrak{H}_{0}$ such that $\mathcal{M}_{n}(g \mathcal{O})=Q(g) \mathcal{M}_{n}(\mathcal{O}) Q(g)^{-1}$, $g \in \mathrm{SU}(2,2)$. Further $Q \widetilde{\mathcal{P}_{+}^{\uparrow}}$ is strongly continuous and the generators of the space time translations satisfy the spectrality condition.

Proof: The properties of isotony, causality and additivity follow directly from the corresponding properties of the net of abstract $\mathrm{C}^{*}$-algebras $\mathcal{O} \mapsto \mathcal{A}_{n}(\mathcal{O})$ in Theorem 3.6. Further recall that for the Bose resp. the Fermi case the one-particle Hilbert space associated to the natural Fock 
representations is $\mathfrak{H}_{n,+}^{\prime} \oplus \mathfrak{H}_{n,-}^{\prime}$ and since $W_{1} \oplus W_{2}$ given above is unitary on it we have from the invariance of the Fock state and Theorem 5.7 that for suitable $g \in \mathrm{SU}(2,2)$ and $\mathcal{O} \in \mathcal{K}$

$$
\mathcal{M}_{n}(g \mathcal{O})=\left(\pi_{0} \circ \alpha_{g}\left(\mathcal{M}_{n}(\mathcal{O})\right)\right)^{\prime \prime}=\left(Q(g) \pi_{0}\left(\mathcal{M}_{n}(\mathcal{O})\right) Q(g)^{-1}\right)^{\prime \prime}=Q(g) \mathcal{M}_{n}(\mathcal{O}) Q(g)^{-1}
$$

Here $Q(g)$ is the second quantization of $W_{1} \oplus W_{2}$ on the symmetric resp. antisymmetric Fock space over $\mathfrak{H}_{n,+}^{\prime} \oplus \mathfrak{H}_{n,-}^{\prime}$. Further, $Q(g) \Omega=\Omega$, where $\Omega$ is the Fock vacuum and $Q \uparrow \mathbb{R}^{4}$ satisfies the spectrum condition because by Theorem 5.5 $\left(W_{1} \oplus W_{2}\right)\left|\mathbb{R}^{4}=\left(V_{1}^{\prime} \oplus V_{2}^{\prime}\right)\right| \mathbb{R}^{4}$ does.

For unbounded regions one defines the corresponding localized von Neumann algebras by additivity.

We will conclude this section mentioning some standard algebraic results for these models that are consequence of the conformal covariance showed above. We will freely use in the following definitions and results from [13, Section 2], [31, Section 4] and 32] (cf. also with references cited therein). Denote by $K_{1}$ the double cone of radius 1 and centered at the origin, by $\mathcal{W}_{r}:=\{x \in$ $\left.\mathbb{R}^{4}|| x_{0} \mid<x_{3}\right\}$ the right wedge and by $\mathcal{V}_{+}$the forward light cone. Recall that there are elements of the conformal group that map these regions in each other. Then we have:

(i) The von Neumann algebras $\mathcal{M}\left(K_{1}\right), \mathcal{M}\left(\mathcal{W}_{r}\right)$ and $\mathcal{M}\left(\mathcal{V}_{+}\right)$are spacially isomorphic and in particular Type $\mathrm{III}_{1}$-factors.

(ii) The modular groups of the von Neumann algebras $\mathcal{M}\left(K_{1}\right), \mathcal{M}\left(\mathcal{W}_{r}\right)$ and $\mathcal{M}\left(\mathcal{V}_{+}\right)$act geometrically.

(iii) Implementation of the PCT transformation using the modular conjugation associated to the von Neumann algebra $\mathcal{M}(\mathcal{W})$ for a wedge region $\mathcal{W}$.

(iv) Essential duality and timelike duality for the forward/backward cones hold.

\section{Conclusions}

We have seen in this paper that the notion of free net (which avoids the explicit use of quantum fields) is particularly well adapted in the massless case for proving standard properties expected for these models, in particular for showing covariance under the conformal group. Further, free

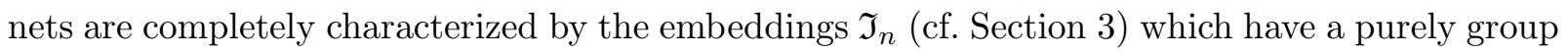
theoretical interpretation.

One possible extension of the massless free net construction is to consider higher dimensional (flat) Minkowski space (although it is not clear that this would be physically meaningful). In 
any case some of the important features of the construction presented here still appear in higher dimensions. Indeed, in a recent paper by Angelopoulos and Laoues [3] with the suggestive title "Masslessness in n-dimensions" it is shown that some of the characteristic group theoretical aspects of the 4-dimensional theory are still valid for $n \geq 5$. In particular, the notion of massless representations (which are again induced representations) can be naturally stated in this context and it is still true that they extend to unitary representations of the corresponding conformal group. A new aspect of higher dimensions though is the fact that the degeneracy of the inducing representations of the associated little groups $\mathcal{E}(n-2)$ affects also its 'rotational' part. Thus generalizing the notion of covariant representation to this situation we conclude that the reduction of the degrees of freedom mentioned in Sections 1 and 3 will be even more present in higher dimensions.

Acknowledgements It is a pleasure to thank Sergio Doplicher and Roberto Longo for their hospitality at the Mathematics Departments of the universities of Rome 'La Sapienza' and 'Tor Vergata', respectively. The visit was supported by a EU TMR network "Implementation of con-

cept and methods from Non-Commutative Geometry to Operator Algebras and its applications", contract no. ERB FMRX-CT 96-0073. I would also like to acknowledge useful conversations with Roberto Longo and Wolfgang Junker.

\section{References}

[1] E. Angelopoulos and M. Flato, On the unitary implementability of conformal transformations, Lett. Math. Phys. 2 (1978), 405-412.

[2] E. Angelopoulos, M. Flato, C. Fronsdal, and D. Sternheimer, Massless particles, conformal group and De Sitter universe, Phys. Rev. D 23 (1981), 1278-1289.

[3] E. Angelopoulos and M. Laoues, Masslessness in n-dimensions, Rev. Math. Phys. 10 (1998), 271-299.

[4] H. Araki, Bogoljubov automorphisms and Fock representations of canonical anticommutation relations, In Operator Algebras and Mathematical Physics, (Proceedings of the summer conference held at the University of Iowa, 1985), P.E.T. Jorgensen and P.S. Muhly (eds.), American Mathematical Society, Providence, 1987.

[5] M. Asorey, L.J. Boya, and J.F. Cariñena, Covariant representations in a fibre bundle framework, Rep. Math. Phys. 21 (1985), 391-404. 
[6] A.O. Barut and R. Rạczka, Properties of non-unitary zero mass induced representations of the Poincaré group on the space of tensor-valued functions, Ann. Inst. H. Poincaré 17 (1972), 111-118.

[7] — Theory of Group Representations and Applications, Polish Scientific Publishers, Warszawa, 1980.

[8] H. Baumgärtel, Operatoralgebraic Methods in Quantum Field Theory. A Series of Lectures, Akademie Verlag, Berlin, 1995.

[9] H. Baumgärtel, M. Jurke, and F. Lledó, On free nets over Minkowski space, Rep. Math. Phys. 35 (1995), 101-127.

[10] H. Baumgärtel and M. Wollenberg, Causal Nets of Operator Algebras. Mathematical Aspects of Algebraic Quantum Field Theory, Akademie Verlag, Berlin, 1992.

[11] H.J. Borchers, Einstein's principle of maximal speed in classical and quantum physics, In Mathematical Physics towards the 21st Century, R.N. Sen and A. Gersten (eds.), Ben-Gurion University of the Negev Press, Beer-Sheva, 1994.

[12] L.J. Boya, J.F. Cariñena, and M. Santander, On the continuity of the boosts for each orbit, Commun. Math. Phys. 37 (1974), 331-334.

[13] R. Brunetti, D. Guido, and R. Longo, Modular structure and duality in conformal quantum field theory, Commun. Math. Phys. 156 (1993), 201-219.

[14] D. Buchholz, Collision theory for massless fermions, Commun. Math. Phys. 42 (1975), 269279.

[15] — Collision theory for massless bosons, Commun. Math. Phys. 52 (1977), 147-173.

[16] D. Buchholz, S. Doplicher, G. Morchio, J.E. Roberts, and F. Strocchi, A model of charges of electromagnetic type, In Operator Algebras and Quantum Field Theory (Proceedings, Rome, July 1-6, 1996), S. Doplicher et al. (ed.), International Press, Boston, 1997.

[17] D. Buchholz and R. Haag, The quest for understanding in relativistic quantum physics, to appear in J. Math. Phys., 2000.

[18] A.L. Carey, Induced representations, reproducing kernels and the conformal group, Commun. Math. Phys. 52 (1977), 77-101. 
[19] A. Einstein, Über einen die Erzeugung und Verwandlung des Lichtes betreffenden heuristischen Gesichtspunkt, Annalen der Physik 17 (1905), 132-148.

[20] _ _ Zur Elektrodynamic bewegter Körper, Annalen der Physik 17 (1905), 891-921.

[21] _ _ Über die Entwicklung unserer Anschauungen über das Wesen und die Konstitution der Strahlung, Phys. Z. 10 (1909), 817-826.

[22] H. Grundling, Systems with outer constraints. Gupta-Bleuler electromagnetism as an algebraic field theory, Commun. Math. Phys. 114 (1988), 69-91.

[23] H. Grundling and C.A. Hurst, Algebraic quantization of systems with a gauge degeneracy, Commun. Math. Phys. 98 (1985), 369-390.

[24] _ A note on regular states and supplementary conditions, Lett. Math. Phys. 15 (1988), 205-212, [Errata: ibid. 17, 173-174 (1989)].

[25] H. Grundling and F. Lledó, Local quantum constraints, submitted to Rev. Math. Phys.

[26] R. Haag, Local Quantum Physics, Springer Verlag, Berlin, 1992.

[27] R. Haag and D. Kastler, An algebraic approach to quantum field theory, J. Math. Phys. 5 (1964), 848-861.

[28] D. Han and Y.S. Kim, Little group for photons and gauge transformations, Am. J. Phys. 49 (1981), 348-351.

[29] S. Helgason, Differential Geometry, Lie Groups, and Symmetric Spaces, Academic Press, New York, 1978.

[30] P. D. Hislop, The modular structure of local algebras associated with massless free quantum fields, Ph.D. thesis, University of California, Berkeley, 1984.

[31] P.D. Hislop, Conformal covariance, modular structure, and duality for local algebras in free massless quantum field theories, Ann. Phys. 185 (1988), 193-230.

[32] P.D. Hislop and R. Longo, Modular structure of local algebras associated with the free massless scalar field theory, Commun. Math. Phys. 84 (1982), 71-85.

[33] H.P. Jakobsen and M. Vergne, Wave and Dirac operators, and representations of the conformal group, J. Func. Anal. 24 (1977), 52-106. 
[34] M. Jammer, Der Begriff der Masse in der Physik, Wissenschaftliche Buchgesellschaft, Darmstadt, 1981.

[35] H.A. Kastrup, Zur physikalischen Deutung und darstellungstheoretischen Analyse der konformen Transformationen von Raum und Zeit, Annalen der Physik 9 (1962), 388-428.

[36] H.A. Kastrup and D.H. Mayer, On some classes of solutions of the wave equation $\partial_{t}^{2} f-\triangle f=$ 0, J. Math. Phys. 11 (1970), 1041-1047.

[37] Y.S. Kim and E.P. Wigner, Cylindrical group and massless particles, J. Math. Phys. 28 (1987), 1175-1179.

[38] R.A. Kunze, Positive definite operator-valued kernels and unitary representations, In Proceedings of the Conference in Functional Analysis, Irvine, 1966, B. Gelbaum (ed.), Academic Press, Boston, 1967.

[39] N.P. Landsman, Induced representations, gauge fields and quantization on homogeneous spaces, Rev. Math. Phys. 4 (1992), 503-527.

[40] N.P. Landsman and U.A. Wiedemann, Massless particles, electromagnetism and Rieffel induction, Rev. Math. Phys. 7 (1995), 923-958.

[41] W. Langbein, Zur physikalischen Interpretation manifest kovarianter Darstellungen der inhomogenen Lorentzgruppe zur Masse Null, Commun. Math. Phys. 5 (1967), 73-87.

[42] F. Lledó, A family of examples with quantum constraints, Lett. Math. Phys. 40 (1997), $223-234$.

[43] _ Algebraic properties of massless free nets, Ph.D. thesis, University of Potsdam, 1999.

[44] _ Massless relativistic wave equations and quantum field theory, in preparation.

[45] G. Mack and I. Todorov, Irreduciblility of the ladder representations of $U(2,2)$ when restricted to the Poincaré subgroup, J. Math. Phys. 10 (1969), 2078-2085.

[46] G.W. Mackey, The Theory of Unitary Group Representations, The University of Chicago Press, Chicago, 1976.

[47] J. Manuceau, M. Sirugue, D. Testard, and A. Verbeure, The smallest $\mathrm{C}^{*}$-algebra for canonical commutations relations, Commun. Math. Phys. 32 (1973), 231-243. 
[48] U.H. Niederer and L. O'Raifeartaigh, Realizations of the unitary representations of the inhomogeneous space-time groups I, Fortschr. Phys. 22 (1974), 111-129.

[49] _ Realizations of the unitary representations of the inhomogeneous space-time groups II, Fortschr. Phys. 22 (1974), 131-157.

[50] V.B. Petkova, G.M. Sotkov, and I.T. Todorov, Local field representations of the conformal group and their physical interpretation, In Supermanifolds, Geometrical Methods and Conformal Groups, H.D. Doebner et al. (ed.), World Scientific, Singapore, 1989.

[51] N. Shnerb and L.P. Horwitz, Gauge and group properties of massless fields in any dimension, J. Phys. A: Math. Gen. 27 (1994), 3565-3574.

[52] D.J. Simms, Lie Groups and Quantum Mechanics, Springer Verlag, Berlin, 1968.

[53] S. Sternberg, Group Theory and Physics, Cambridge University Press, Cambridge, 1995.

[54] R.F. Streater and A.S. Wightman, PCT, Spin and Statistics, and All That, Addison-Wesley, Redwood City, 1989.

[55] I.T. Todorov, M.C. Mintchev, and V.B. Petkova, Conformal invariance in quantum field theory, Publ. Scuola Normale Superiore di Pisa, Classe di Scienze (1978), 1-373.

[56] G. Warner, Harmonic Analysis on Semi-Simple Lie Groups I, Springer-Verlag, Berlin, 1972.

[57] S. Weinberg, Feynman rules for any spin. II. Massless particles, Phys. Rev. 134 (1964), B882-B896.

[58] _ The quantum theory of massless particles, In Lectures on Particle and Field Theory (Brandais Summer Institute in Theoretical Physics 1964, vol. II ), S. Deser and K.W. Ford (eds.), Prentice Hall Inc., Englewood Cliffs, 1965.

[59] E.P. Wigner, On unitary representations of the inhomogeneous Lorentz group, Ann. Math. 40 (1939), 149-204. 